Old Dominion University

ODU Digital Commons

Mathematics \& Statistics Theses \&

Dissertations

Mathematics \& Statistics

Spring 1987

\title{
On a Moving Boundary Problem of Transitional Ballistics
}

Jen-Ing G. Hwang

Old Dominion University

Follow this and additional works at: https://digitalcommons.odu.edu/mathstat_etds

Part of the Applied Mathematics Commons, and the Fluid Dynamics Commons

\section{Recommended Citation}

Hwang, Jen-Ing G.. "On a Moving Boundary Problem of Transitional Ballistics" (1987). Doctor of Philosophy (PhD), Dissertation, Mathematics \& Statistics, Old Dominion University, DOI: 10.25777/5zaker78

https://digitalcommons.odu.edu/mathstat_etds/78

This Dissertation is brought to you for free and open access by the Mathematics \& Statistics at ODU Digital Commons. It has been accepted for inclusion in Mathematics \& Statistics Theses \& Dissertations by an authorized administrator of ODU Digital Commons. For more information, please contact digitalcommons@odu.edu. 


\title{
ON A MOVING BOUNDARY PROBLEM OF TRANSITIONAL BALLISTICS
}

\author{
by \\ Jen-Ing G. Hwang \\ B.S. June 1980, Fu Jen Catholic University, Taiwan, R.o.C. \\ M.S. May 1984, Old Dominion University \\ A Dissertation Submitted to the Faculty of \\ old Dominion University in Partial Fulfillment of the \\ Reguirements for the Degree of \\ DOCTOR OF PHIIOSOPHY \\ COMPUTATIONAL AND APPLIED MATHEMATICS
}

OLD DOMINION UNIVERSITY

May 1987

Approved by

Dr. Charlie H. Cooke, (Director)

Reproduced with permission of the copyright owner. Further reproduction prohibited without permission. 
TO MY PARENTS

ii

Reproduced with permission of the copyright owner. Further reproduction prohibited without permission. 


\section{ACKNOWLEDGEMENTS}

I wish to express appreciation to my advisor, Dr. Charlie H. Cooke, for his guidance, patience, and assistance during my doctoral research. I would also like to thank the other members of my dissertation committee, Drs. John M. Dorrepaal and Surendra N. Tiwari, for their helpful suggestions and comments on my dissertation.

To the faculty and graduate students in the Mathematics Department, I extend a sincere thanks for the kindness shown to me. In particular, the understanding and helpfulness of the Department Chairman, Dr. John Tweed, and the Graduate Program Director, Dr. Philip R. Wohl, are deeply appreciated.

I consider myself fortunate to have had friends who always gave me emotiona? support and encouragement during my graduate studies, for which I am grateful. I am especially grateful to my best friend, Bao-Iing Tsay, for her constant encouragement, concern, and assistance.

A special word of thanks is reserved for my parents, without whose continual support, encouragement, and love, this study would not have been possible.

Finally, I wish to thank the Mathematics Department Secretary, Barbara Jeffrey, for her assistance during my graduate studies and her excellent typing of this manuscript. 
IIST OF TABLES . . . . . . . . . . . . . vi vi

LIST OF FIGURES . . . . . . . . . . . . . vii

Chapter

1. INTRODUCTION ............... 1

1.1 Basic Literature Survey . . . . . 2

1.2 Establishment of Need ....... . 3

1.3 Statement of Purpose . . . . . . 4

2. BACKGROUND MATERIAL AND

PRELIMINARY CONSIDERATIONS . . . . . 7

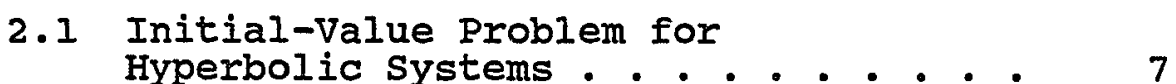

The Riemann Problem;
Matrix Splitting........ . 12

2.3 Godunov's Method .......... 14

3. SOME UPWIND BIASED DIFFERENCE SCHEMES . . . 22

3.1 The WDF Form . . . . . . . . 24

3.2 Roe's scheme........... . 29

4. A MOVING BOUNDARY PROBLEM OF TRANSITIONAL BALLISTICS . . . . . . . . . . 39

4.1 A One-Dimensional Model ...... 41

4.1.1 Algorithm Overview ..... 43

4.1.2 Governing Equations of
Idealized Compressible Flow . . 46

4.1.3 A Local Finite Volume Scheme - 47

4.1.4 Axisymmetric Problems ..... 5].

4.2 Numerical Validation of the Expanding 
TABLE OF CONTENTS-continued

Page

5. SUPERSONIC PROJECTILE MOTION . . . . . . 58

5.1 The Euler Equations . . . . . . . 58

5.2 Eigenvector Projection . . . . . 59

5.3 An Operator-Split,
TVD Algorithm . . . . . . . . . 66

5.4 Weapons Nomenclature . . . . . . 68

5.5 Numerical Results;

Supersonic Moving Projectile : . . . 70

6. A PHYSICALIY MOTIVATED WALL BOUNDARY TREATMENT . . . . . . . . . 78

6.1 Rarefaction Waves ......... 79

6.2 Compression Waves . . . . . . . . 83

7. SUMMARY AND CONCLUSIONS ........ 87

REFERENCES . . . . . . . . . . . . . . 89

AUTOBIOGRAPHICAI STATEMENT . . . . . . . . . 92 


\section{LIST OF TABLES}

\section{Table}

Page

5.1 Comparison of stagnation Point

Properties . . . . . . . . . . . . . . . 
2.1 Characteristics and constant state Solution of the Riemann Problem . . . . . .

4.1 Typical Weapons Configuration Axisymmetric Geometry . . . . . . . . . . 42

4.2 Algorithm Schematic . . . . . . . . . . 44

4.3 Cell Division . . . . . . . . . . . 45

4.4 Phase Plane . . . . . . . . . . . . . 48

4.5 Pressure Comparison . . . . . . . . . 53

4.6 Density Comparison . . . . . . . . . . 54

4.7 Velocity Comparison for Numerical

Wall Boundary Condition . . . . . . . . 55

4.8 Velocity Comparison; Theoretical

Wall Boundary Condition . . . . . . . . . . 57

5.1 Gun Blast Mach Contours (50.0 us) . . . . . 69

5.2 Gun Blast Density Contours (50.0 $\mu \mathrm{s})$. . . . 71

5.3 Velocity Vector Plot $(50.0 \mu \mathrm{s})$. . . . . . 72

5.4 Gun Blast Mach Contours (100.0 us) . . . . 73

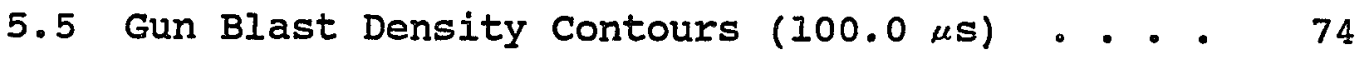

5.6 Velocity Vector Plot $(100.0 \mu \mathrm{s})$. . . . . 75

6.1 Schematic of Rarefaction Waves . . . . . . 82 
ABSTRACT

ON A MOVING BOUNDARY PROBLEM OF TRANSITIONAL BALIISTICS

\author{
Jen-Ing G. Hwang \\ old Dominion University, 1987 \\ Director: Dr. Charlie H. Cooke
}

\begin{abstract}
A major problem which arises in computer simulation of the firing of a gun weapon is the development of numerical schemes which effectively account for the physics of projectile motion. The chief difficulty is that away from the projectile the calculation is ordinarily accomplished on a fixed numerical grid, whereas due to projectile movement some cells of the grid near the projectile undergo volume changes as the calculation proceeds. A local finite volume scheme is developed which accounts for the expansion or compression of cells fore-and-aft of the projectile. Through the process of numerical experiment, the effectiveness of the scheme is assessed, with quite good results.

The rapid discharge of propellent gas from a gun
\end{abstract} weapon produces a strong shock wave which propagates into the environment, while other interacting shocks form within the developing plume. For this reason, strong interest in the determination of shock capturing algorithms which can be used away from the projectile arises. In this respect, a theoretical weak derivative form (WDF) is derived for linear hyperbolic systems of conservation laws. The virtue of the WDF approach is that 
it indicates how to difference in the presence of a flow discontinuity, without differencing across the discontinuity. This differencing produces a robust shock capturing scheme whose extension to the nonlinear case is apparent.

The WDF shock capturing scheme so obtained is shown to be equivalent to a flux-splitting scheme studied by P.I. Roe, thus leading to a better understanding of the schemes of Godunov and Roe, as well as upwind differencing in general. Roe's scheme is investigated in detail. Three views of the scheme are obtained, one of which is new. Harten's second-order accurate extension of Roe's method is then used in simulating the flow around a typical weapon's configuration. These numerical results reinforce the belief that the local finite volume scheme effectively accounts for projectile motion. 


\section{Chapter 1}

\section{INTRODUCTION}

Transitional ballistics concerns the analysis of a weapon's firing, during that short time interval (a few milliseconds) in which the bullet exits the breech and traverses a short distance (a few calibres) downstream. Useful information concerning near-field blast overpressures can be obtained from flow simulations which do not include the effects of projectile movement. However, such effects can not be ignored in stress analyses concerned with the design of muzzle brakes and weapons silencers. A problem which has been of some interest over the past five years has been the development of numerical schemes which effectively account for the physics of the projectile motion during the flow calculation. obviously, one would not wish to treat the moving projectile (in an airfoil fashion) by use of a coordinate system fixed to the projectile. Hence, there arises the need for special treatment of computational cells fore-and-aft of the projectile, which expand or compress in size as the calculation progresses, while a fixed grid possessing constant volume cells still remains useful away from the vicinity of the projectile.

The rapid discharge of a propellant gas from a weapon produces a strong shock wave which propagates into the environment. Quite aside from the problem of accounting 
for projectile motion is the difficulty of effectively capturing the interacting shocks and contact surfaces which form within the developing plume. For this reason, strong interest in shock capturing algorithms which can be used away from the projectile is a concomitant facet of the problem under consideration.

\subsection{Basic Literature survey}

Numerical analyses of the transitional ballistics problem have proceeded in stages characterized by increasingly complex models. As it is known that good pressure approximations can be obtained from the Euler equations of ideal compressible flow, no general usage of Navier-stokes models has occurred. Moretti [I]* has studied the effects of the precursor flow, through use of complex mapping techniques which simplify the exterior computational domain, and by employing one-dimensional modelling of flow within the breech. With the projectile omitted, typical estimates of blast wave overpressure have been obtained through numerical calculation of flow from the open end of a shock tube [2]. More realistic simulations which include the bullet and muzzle brake in the model have also been performed $[3,4]$.

\footnotetext{
*The numbers in the brackets indicate references.
} 
Two basically different approaches to simulation of projectile motion have emerged: Widhopf and Buell [3] have used the Godunov method (away from the bullet) together with a physically motivated, heuristic algorithm which accounts for the cell expansion and compression adjacent to the projectile. The sequence of operations proposed in [3] has been modified by Fansler [5], in the interest of alleviating a non-physical pressure buildup on the rear of the projectile. Carofano [4] uses the second-order accurate shock capturing method of Harten [6] at cells away from the bullet. To avoid the problem of cells whose size can vary, he calculates near the projectile on a uniform grid which moves with the projectile. The chief drawback of his scheme results from a loss of accuracy when interpolating back and forth between the fixed and moving grids.

\subsection{Establishment of Need}

As has been established, the observed weaknesses in present schemes which are designed to account for projectile motion declare a need for further research on this problem; one weakness being interpolation error, and the other a non-physical pressure buildup. This non-physical pressure buildup is assumed to result from an incorrect physical model, as we now make more clear. The first stage in the Widhopf scheme is intuitive: The cell adjacent to the projectile is expanded; then the volume 
increase is accounted for by an isenthalpic (Joule) expansion process on the fluid in this cell. In the next stage of flow update, pressure work is accounted for by a suitable modification of the energy equation. However, one might expect, in analogy with the withdrawing piston problem, that flow adjacent to the back of the projectile encounters an isentropic, rather than an isenthalpic, expansion process.

\subsection{Statement of Purpose}

one aim of this research is to develop, implement, and assess the effectiveness of a projectile movement technique which correctly accounts for the physics involved. Our contribution here, using methods which are mathematically rigorous as well as aesthetically pleasing, is the development of a local finite volume scheme for treatment of cells whose volumes vary over a time step. Away from the vicinity of the bullet, quite general numerical methods could be used in conjunction with this scheme.

A second purpose of this thesis is to investigate equivalences or similarities between certain shock capturing schemes used for calculating numerical solutions of the Euler Equations. Investigations of this type aid in understanding the properties of such schemes and in the synthesis of new schemes. Such previous investigation has been done by Harten [7], where it is shown that, in the 
linear case, the shock capturing schemes of Godunov [8], Roe $[9,10]$ and Courant-Isaacson-Rees [7] are equivalent to each other, as well as equivalent to a flux-splitting scheme [11]. Similarities between the schemes of Godunov, Roe and Osher [12] are studied in [13]. It is proposed here to continue the study of analogies between shock capturing schemes to the nonlinear case. Particularly, attention is focused on the Roe scheme, as compared to the flux-splitting schemes developed in [11]. For the nonlinear case it is established that Roe's scheme is, in reality, also a flux-splitting, with the split flux matrices evaluated in a particular way.

Chapter 2 contains background material relevant to an understanding of later research. The Riemann problem for linear hyperbolic systems is presented, and the properties of Riemann invariants are pointed out. The Godunov shock capturing method is illustrated, along with its salient desirable properties, and matrix splitting is introduced.

In Chap. 3 the properties of Riemann invariants are used to establish a weak derivative form (WDF) for linear hyperbolic systems. The importance of the WDF form is that it shows precisely how to difference at a discontinuity, without differencing across the discontinuity. This differencing leads to a robust shock capturing scheme whose extension to the nonlinear case is readily apparent. It is shown that this scheme is identical with a certain scheme of Roe. Several upwind 
biased schemes which differ in the nonlinear case, but which reduce to a single scheme in the linear case, are investigated. In so doing, three views of Roe's scheme, one of which is new, are obtained.

In chap. 4 a local finite volume scheme which correctly embodies the physics of projectile movement is derived. The upwind scheme formulated by resort to the WDF form is then used for calculations which verify the effectiveness of the local finite volume scheme, by employing a shock tube flow problem whose solution is well-known. Comparison of results shows a very favorable performance for the scheme, at cells behind the projectile.

In Chap. 5 the problem of a bullet moving at supersonic speed through the chamber of a weapon's silencer attached to a gun weapon is considered. This flow is calculated by employing the local finite volume scheme near the projectile. Here, knowledge of how the bow shock should move in front of the projectile allows verification of accuracy for the calculation. Efficacy of the method is re-enforced by the quality of these results. Finally, in chap. 6 the problem of boundary condition treatment for flows characterized by moving shocks which can strike a wall boundary is discussed. A boundary treatment which automatically accounts for local wave behavior is reiterated. 
BACKGROUND MATERIAL AND PRELIMINARY CONSIDERATIONS

In the present chapter there is reviewd some basic mathematical concepts which provide a foundation for later research. The focus of interest is a form of the initial-value problem for hyperbolic systems, called the Riemann problem. In addition, Godunov's method [8] is introduced, and for linear systems the connection between this method and the method of flux splitting [1I] is established.

2.1. Initial-Value Problem for Hyperbolic Systems The initial-value problem for hyperbolic system of conservation laws has form

$$
\begin{aligned}
& \frac{\partial \bar{U}}{\partial t}+\frac{\partial F(\bar{U})}{\partial x}=0 \\
& \bar{U}(x, 0)=\bar{U}_{0}(x), \quad-\infty<x<\infty
\end{aligned}
$$

Here $\bar{U}(x, t)$ is a column vector of $N$ unknowns, and the flux function $F(\bar{U})$ is a vector valued function of $N$ components. Equation (2.1a) can be written in matrix form as 


$$
\frac{\partial \bar{U}}{\partial t}+A(\bar{U}) \frac{\partial \bar{U}}{\partial X}=0, \quad A(\bar{U})=\frac{\partial F(\bar{U})}{\partial \bar{U}}
$$

where $A(\bar{U})$ is the Jacobian matrix. The system (2.1) is called hyperbolic if all eigenvalues of $A(\bar{U})$ are real and $A(\bar{U})$ possesses a complete set of eigenvectors. It is assumed that the eigenvales $\left\{\lambda_{i}(\bar{U})\right\}_{i=1}^{\mathbb{N}}$ are distinct, nonzero, and arranged in an increasing order,

$$
\lambda_{1}<\lambda_{2}<\ldots<\lambda_{m}<0<\lambda_{m+1}<\ldots<\lambda_{N}
$$

with corresponding right eigenvectors $\left\{R_{i}(\bar{U})\right\}_{i=1}^{N}$. Let

$$
M(\bar{U})=\left(R_{1}(\bar{U}), R_{2}(\bar{U}), \ldots, R_{N}(\bar{U})\right)
$$

be a matrix with $R_{i}(\bar{U})$ as column $i$. Then, $\mathbb{M}^{-1}(\bar{U})$ has rows $\left\{I_{i}(\bar{U})\right\}_{i=1}^{N}$ which constitute a complete system of left eigenvectors of $A(\bar{U})$. Consequently,

$$
I_{i} R_{j}=\delta_{i j}
$$

and 


$$
\mathrm{M}^{-1} \mathrm{AM}=\Lambda
$$

where

$$
\Lambda_{i j}=\lambda_{i} \delta_{i j}
$$

To allow for discontinuous behavior, one admits weak solutions which satisfy system (2.1) in the sense of distribution theory; i.e.

$\int_{0}^{\infty} \int_{-\infty}^{\infty}\left[W_{t} \bar{U}+W_{x} F(\bar{U})\right] d x d t+\int_{-\infty}^{\infty} w(x, 0) \bar{U}(x, 0) d x=0$

for all $c^{\infty}$ test functions $W(x, t)$ which vanish for large $|x|+|t|$. Thus, a piecewise smooth $\bar{U}(x, t)$ is a weak solution of (2.1) if and only if

(i) $\overline{\mathrm{U}}$ satisfies (2.1a) pointwise in each smooth region.

(ii) Across each curve of discontinuity the Rankine-Hugoniot relation

$$
[F(\bar{U})]=S[\bar{U}]
$$

holds, where $s=\frac{d x}{d t}$ is the speed of propagation of the discontinuity, and [ ] is the 
conventional notation for jumps. In the case

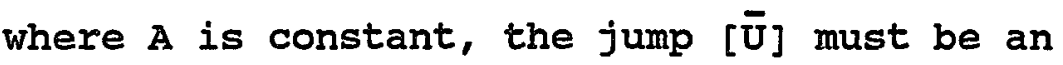
eigenvector of $A[14]$, with $S$ the corresponding eigenvalue, and discontinuities in the initial data $\bar{U}_{0}(x)$ must propagate along characteristic curves $c: \frac{d x}{d t}=\lambda_{i}$.

The characteristic curves were originally analyzed in investigations of the cauchy initial-value problem. At each point of a characteristic the equation

$$
\left(\lambda_{i} I-A\right) \bar{U}_{x}=\frac{d \bar{U}}{d t}
$$

is characterized by nonunique solutions. Thus, certain compatibility conditions arise, from the requirement that

$$
\operatorname{Rank}\left(\lambda_{i} I-A\right)=\operatorname{Rank}\left[\lambda_{i} I-A \mid \frac{d \bar{U}}{d t}\right]
$$

In many circumstances, and particularly when $A$ is a constant matrix, the compatibility relations (2.9) can be integrated along characteristics to produce the so-called Riemann invariants of eqn. (2.1). A Riemann invariant is a scalar function $V_{i}(\bar{U})$ which is constant on the 
corresponding characteristic $c_{i}: \frac{d x}{d t}=\lambda i$. For $\bar{U}$ a strong solution, an equivalent definition is that scalar function $V_{i}(\bar{U})$ is a Riemann invariant if $\frac{\partial V_{j}}{\partial \bar{U}}$ is an eigenvector of $A^{T}[15]$, i.e. $\frac{\partial V_{j}}{\partial \bar{U}}=\left[I_{i}(\bar{U})\right]^{T}$. Clearly, for constant $A$ and strong $\bar{U}$ this implies each scalar function

$$
v_{i}(\bar{U})=\bar{U}^{-T} L_{i} T, \quad i=1,2, \ldots, N
$$

is a Riemann invariant of eqn. (2.1).

From eqns. (2.8) and (2.9) we infer that classical

derivations of Riemann invariants depend upon the solution being differentiable. However, from such arguments a Riemann invariant can be guaranteed constant along a characteristic only in regions where the solution is smooth. In general, this is as much as can be said, since for ideal compressible flow the Riemann invariants are well known to exhibit discontinuous behavior across both shocks and contact discontinuities [16]. However, if the matrix $A$ of eqn. (2.1c) is constant, through use of the weak solution concept, eqn. (2.6), one can establish that the Riemann invariants $(2.10)$ remain constant across jump discontinuities in solutions that are piecewise smooth [17]. 


\subsection{The Riemann Problem; Matrix Splitting}

The Riemann problem for system (2.1) occurs when the initial data is only piecewise smooth, i.e.,

$$
\begin{aligned}
& \frac{\partial \bar{U}}{\partial t}+A(\bar{U}) \frac{\partial \bar{U}}{\partial x}=0 \\
& \bar{U}(x, 0)= \begin{cases}\bar{U}_{L}(x), & x \leq x_{0} \\
\bar{U}_{R}(x), & x>x_{0}\end{cases}
\end{aligned}
$$

Here $\bar{U}_{R}$ and $\bar{U}_{I}$ are smooth functions, but $\bar{U}(x, 0), \frac{\partial \bar{U}}{\partial x}(x, 0)$ may have a finite jump discontinuity at $x_{0}$. Because of the hyperbolicity assumption, when $A$ is constant the system (2.11a) can be diagonalized by a similarity transformation

$$
\mathrm{V}=\mathrm{M}^{-1} \overline{\mathrm{U}}
$$

The system (2.11a) can be written as a set of decoupled equations

$$
\frac{\partial V}{\partial t}+\Lambda \frac{\partial V}{\partial x}=0
$$

where $\Lambda$ is defined in eqn. $(2.5)$. 
Let $\lambda$ be a constant, and introduce the notation

$$
\begin{aligned}
& \lambda^{+} \equiv \max (\lambda, 0)=\frac{1}{2}(\lambda+|\lambda|) \\
& \lambda^{-} \equiv \min (\lambda, 0)=\frac{1}{2}(\lambda-|\lambda|)
\end{aligned}
$$

Also define the elements of $|\Lambda|$ by

$$
|\Lambda|_{i j}=\left|\lambda_{i}\right| \delta_{i j}
$$

Therefore,

$$
|\Lambda|=\Lambda^{+}-\Lambda^{-}
$$

$$
\Lambda=\Lambda^{+}+\Lambda^{-}
$$

where $\Lambda^{+}{ }_{i j}=\lambda_{i}^{+} \delta_{i j}$ and $\Lambda^{-}{ }_{i j}=\lambda_{i}^{-} \delta_{i j}$.

Also, if one defines

$$
\begin{aligned}
& |A|=M|\Lambda| M^{-1} \\
& A^{+}=M \Lambda^{+} M^{-1} \\
& A^{-}=M \Lambda^{-} M^{-1},
\end{aligned}
$$


then

$$
\begin{aligned}
A & =A^{+}+A^{-} \\
A^{+} & =\frac{1}{2}(A+|A|) \\
A^{-} & =\frac{1}{2}(A-|A|)
\end{aligned}
$$

Equation (2.18a) describes what is called a splitting of the matrix $\mathrm{A}$.

\subsection{Godunov's Method}

The solution to system (2.1) may develop shocks and contact discontinuities, even when the initial data are smooth. The technique of shock capturing has been very successful for computing discontinuous solutions. The main advantage of shock capturing lies in its simplicity. In this technique the discontinuity evolves naturally as part of the computation; no special treatment is required for handling jumps at a shock.

The classical method of Godunov [8] utilizes piecewise constant approximating functions. Discontinuities at each cell interface are resolved through exact solution of a local Riemann problem, which in the most general case is nonlinear and requires 
iterative solution.

By introducing the discrete representation $x_{i}=i . \Delta x$, $t_{n}=n . \Delta t$, and assuming that $\bar{U}_{i}^{n}$ is the average value in the computational cell centered on $x_{i}$ at time $t_{n}$, the Godunov scheme for system (2.1) may be written as

$$
\bar{U}_{i}^{n+1}=\bar{U}_{i}^{n}-\frac{\Delta t}{\Delta x}\left[F_{G}\left(\bar{U}_{i}^{n}, \bar{U}_{i+1}^{n}\right)-F_{G}\left(\bar{U}_{i-1}^{n}, \bar{U}_{i}^{n}\right)\right]
$$

where the numerical flux function $F_{G}\left(\bar{U}_{i}^{n}, \bar{U}_{i+1}^{n}\right)$ is taken to be the flux value arising at $x_{i+1 / 2}=x_{i}+\frac{1}{2} \Delta x$ in the exact solution of a local Riemann problem.

The linear case: $A(U)=$ constant

We find it instructive to describe Godunov's scheme further, for the linear case where $A(\bar{U})$ is constant. At a typical cell interface $x_{i+1 / 2}$, the local Riemann problem can be written:

$$
\begin{gathered}
\frac{\partial \bar{U}}{\partial t}+A \frac{\partial \bar{U}}{\partial x}=0 \\
\bar{U}(x, 0)= \begin{cases}\bar{U}_{I}=\bar{U}_{i}^{n}, & x<x_{i+1 / 2} \\
\bar{U}_{R}=\bar{U}_{i+1}^{n}, & x>x_{i+1 / 2}\end{cases}
\end{gathered}
$$


By use of the Riemann invariants, it can be shown that the solution of system (2.20) is composed of constant states, separated by a fan of $\mathrm{N}$ characteristic lines (see Fig. 2.1).

Let

$$
\begin{array}{ll}
\lambda_{0}=-\infty, & { }^{\lambda}+1=+\infty \\
\bar{U}_{0}=\bar{U}_{L}, & \bar{U}_{\mathrm{N}}=\bar{U}_{R}
\end{array}
$$

and

$$
\begin{aligned}
\bar{U}(x, t)=\bar{U}_{k} \text { for } \lambda_{k}<x / t /<\lambda_{k+1} \\
k=0, I, 2, \ldots, N
\end{aligned}
$$

According to the Rankine-Hugoniot relations, the jumps across the characteristics must satisfy

$$
\left[\bar{U}_{k}\right]=\bar{U}_{k}-\bar{U}_{k-1}=\alpha_{k} R_{k}
$$

for some constant $\alpha_{k}$.

Thus,

$$
\bar{U}_{R}-\bar{U}_{L}=\sum_{j=1}^{N} \alpha_{j} R_{j}
$$

and

$$
\bar{U}_{k}-\bar{U}_{L}=\sum_{j=1}^{k} \alpha_{j} R_{j}, \quad k=1,2, \ldots, N
$$




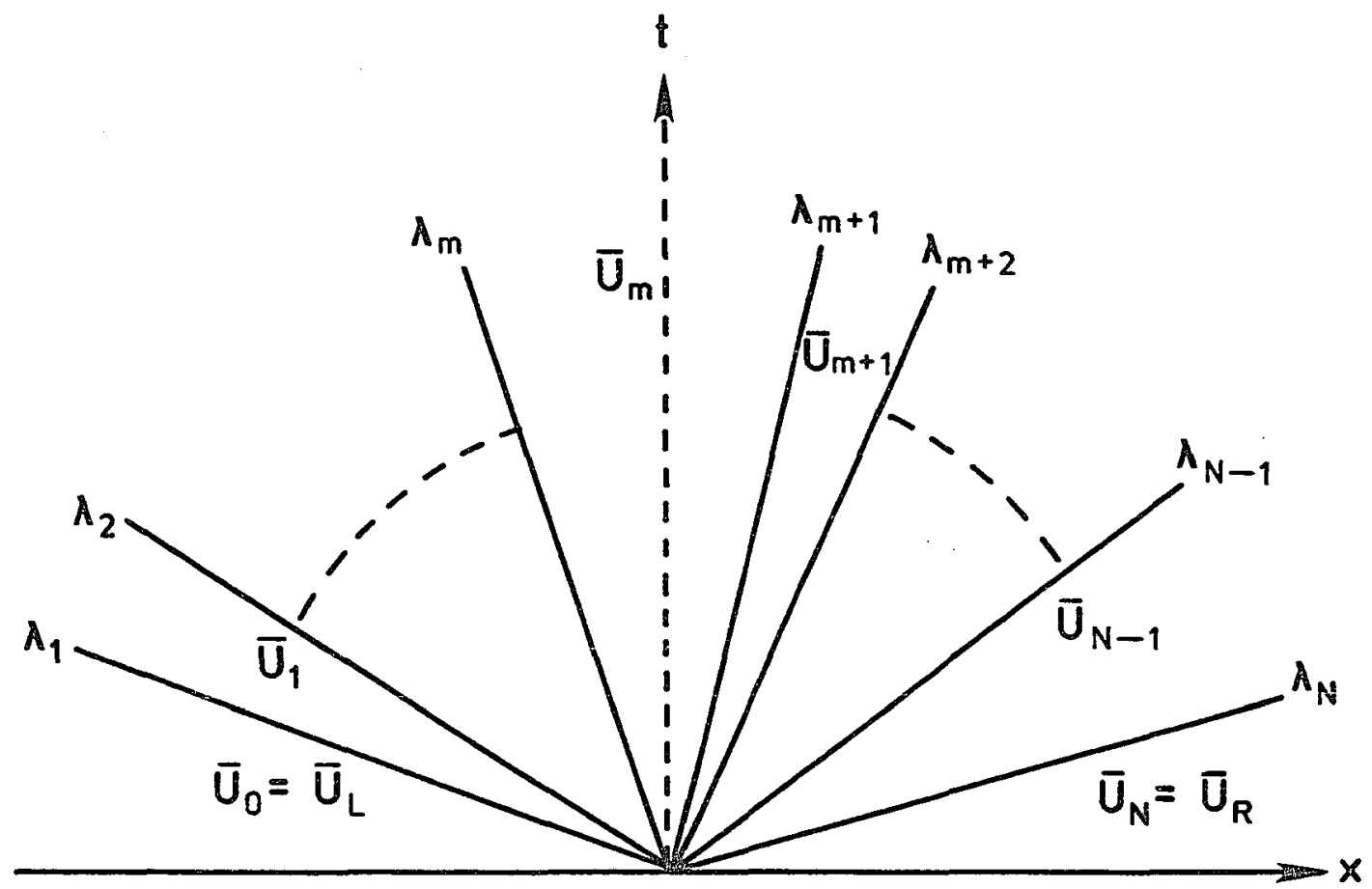

Fig. 2.1 Characteristics and Constant State Solution of the Riemann Problem. 
It should be noted that

$$
\bar{U}_{R}-\bar{U}_{k}=\sum_{j=k+1}^{N} \alpha_{j} R_{j}, \quad k=0,1, \ldots, N-1
$$

Therefore,

$$
2 \bar{U}_{k}=\bar{U}_{L}+\bar{U}_{R}+\sum_{j=1}^{k} \alpha_{j} R_{j}-\sum_{j=k+1}^{N} \alpha_{j} R_{j}
$$

Multiplying by $A$ and picking $\mathrm{k}=\mathrm{m}$, one finds

$$
2 A \bar{U}_{m}=A \bar{U}_{I}+A \bar{U}_{R}+\sum_{j=1}^{m} \alpha_{j} A R_{j}-\sum_{j=m+1}^{N} \alpha_{j} A R_{j}
$$

As the linear flux vector is $F=A \bar{U}$, there emerges

$$
2 F_{G}\left(\bar{U}_{i}^{n}, \bar{U}_{i+1}^{n}\right)=F_{I}+F_{R}+\sum_{j=1}^{m} \alpha_{j}^{\lambda} R_{j}-\sum_{j=m+I}^{N} \alpha_{j} R_{j} R_{j}
$$

where

$$
F_{L}=F\left(\bar{U}_{I}\right)=A \bar{U}_{L}, \quad F_{R}=F\left(\bar{U}_{R}\right)=A \bar{U}_{R}
$$


and where $\mathrm{F}_{G}\left(\overline{\mathrm{U}}_{i}^{\mathrm{n}}, \overline{\mathrm{U}}_{i+1}^{\mathrm{n}}\right)$ is defined in (2.19). By using eqn. (2.2), eqn. (2.27) can be rewritten as

$$
2 F_{G}\left(\bar{U}_{i}^{n}, \bar{U}_{i+1}^{n}\right)=F_{L}+F_{R}-\sum_{j=1}^{N} \alpha_{j}|\lambda j| R_{j}
$$

From eqn. (2.17a), there results

$$
|A| M=M|\Lambda|
$$

i.e.

$$
|A| R_{j}=\left|\lambda_{j}\right| R_{j}, \quad j=1,2, \ldots, N
$$

Hence, eqn. (2.28) becomes

$$
2 F_{G}\left(\bar{U}_{i}^{n}, \bar{U}_{i+1}^{n}\right)=F_{L}+F_{R}-|A| \sum_{j=1}^{N} \alpha_{j} R_{j}
$$

By using eqns. $(2.22)$ and $(2.30)$, it emerges that

$$
F_{G}\left(\bar{U}_{i}^{n}, \bar{U}_{i+1}^{n}\right)=\frac{F_{I}+F_{B}}{2}-\frac{|A|}{2}\left(\bar{U}_{R}-\bar{U}_{L}\right)
$$

Introducing the notation 


$$
\Delta_{i+1 / 2} \bar{U}=\bar{U}_{i+1}^{n}-\bar{U}_{i}^{n}
$$

and noting that $\bar{U}_{I}=\bar{U}_{i}^{n}, \quad \bar{U}_{R}=\bar{U}_{i+1}^{n}$, eqn. (2.3I) becomes

$$
F_{G}\left(\bar{U}_{i}^{n}, \bar{U}_{i+1}^{n}\right)=\frac{F_{i}+F_{i+1}}{2}-\frac{|A|}{2} \Delta_{i+1 / 2} \bar{U}
$$

where $\quad F_{i}=F\left(\bar{U}_{i}^{n}\right)$.

As a result of substituting eqn. (2.33) in eqn. (2.19), one obtains

$$
\begin{aligned}
\overline{\mathrm{U}}_{i}^{\mathrm{n}+1}=\overline{\mathrm{U}}_{i}^{\mathrm{n}}- & \frac{\Delta t}{\Delta \mathrm{x}}\left\{\left[\frac{F_{i}+F_{i+1}}{2}-\frac{|\mathrm{A}|}{2} \Delta_{i+1 / 2} \overline{\mathrm{U}}\right]\right. \\
& \left.-\left[\frac{F_{i-1}+F_{i}}{2}-\frac{|A|}{2} \Delta_{i-1 / 2} \overline{\mathrm{U}}\right]\right\}
\end{aligned}
$$

Equation (2.34) simplifies to

$$
\begin{aligned}
\bar{U}_{i}^{n+1}=\bar{U}_{i}^{n}-\frac{\Delta t}{\Delta x} & {\left[\frac{A}{2} \Delta_{i+1 / 2} \bar{U}-\frac{|A|}{2} \Delta_{i+1 / 2} \bar{U}\right.} \\
& \left.+\frac{A}{2} \Delta_{i-1 / 2} \bar{U}+\frac{|A|}{2} \Delta_{i-1 / 2} \bar{U}\right] .
\end{aligned}
$$


By using eqn. (2.18), Godunov's scheme becomes

$$
\overline{\mathrm{U}}_{i}^{\mathrm{n}+1}=\overline{\mathrm{U}}_{i}^{\mathrm{n}}-\frac{\Delta t}{\Delta \mathrm{x}}\left[\mathrm{A}^{-} \Delta_{i+1 / 2} \overline{\mathrm{U}}+\mathrm{A}^{+} \Delta_{i-1 / 2} \overline{\mathrm{U}}\right]
$$

Thus, in the linear case Godunov's scheme is equivalently obtained by a splitting of the matrix $A$. In the literature this is called a flux splitting [11]. The great advantage of Godunov's method is the clear physical picture from using the Riemann problem. A major drawback of this method is the high cost of solving the Riemann problem exactly for the nonlinear case. Furthermore, all information obtained from the exact solution of the Riemann problem is generally not utilized. Roe [9] considers, as an economical replacement for the exact solution to the nonlinear problem, a local linearization of system (2.1). The linearizing matrix, $\tilde{A}$, is chosen to satisfy certain special properties [10]. In the next chapter, we will discuss and clarify Roe's scheme; as well, his scheme will be derived through an alternate approach. 


\section{Chapter 3 \\ SOME UPWIND BIASED DIFPRRENCE SCHEMES}

A classical approach to derivation of finite difference methods for numerical solution of partial differential equations is to approximate derivatives at each mesh point by truncated Taylor's series. When differentiable functions are involved, this is a viable method of attack. However, hyperbolic systems often possess weak solutions which are only piecewise smooth. A typical example is the occurrence of shock waves in fluid flow modeled by the Euler equations.

For such cases, when a shock wave moves through a mesh point, the Taylor's series method breaks down, as derivatives do not exist at grid points which coincide with the shock position. In the past, research directed to discovery of numerical algorithms which are robust in the presence of discontinuities has proceeded in a somewhat heuristic manner. The Russian mathematician S.K. Godunov, proceeding from a physical standpoint and having knowledge of methods for solving the standard shock tube problem, applied this knowledge in a very clever way to obtain one of the first robust shock capturing algorithms [8]. This method is still widely used in gun blast calculations [3]. The British aeronautical engineer P.I. Roe [9] has more recently shown how to efficiently 
approximate the solution to the nonlinear Riemann problem utilized by Godunov. In a second paper, Roe [10] goes even further, giving a view of several classical finite difference schemes, which when coupled with his first result and applied to the Godunov approach obtains an even better approximation to the fluxes sought after when solving the Riemann problem.

One purpose of this research is to better understand Roe's second scheme. Our approach is to study the relations between this and other upwind biased schemes. In particular, it is shown how to obtain the scheme through an alternative approach.

For linear hyperbolic systems characterized by weak solutions which are piecewise continuous and bounded, there is obtained, rigorously, a weak form of the partial differential equation which holds at mesh points even in the presence of a moving discontinuity. This weak form does not involve the classical sobolev test function space, as no integrals are necessary. This weak equation has a simple, yet elegant merit; it shows explicitly how to difference in the presence of a discontinuity, without fear of differencing across the discontinuity. Moreover, this differencing produces directly the upwind schemes of Godunov [8], Roe $[9,10]$ and the flux-vector splitting of Steger-Warming [11], all of which are known to coincide, for linear hyperbolic systems. Furthermore, the path by which to proceed in the nonlinear case becomes somewhat 
more clear than in the presentation of Roe's second paper $[10]$.

In the first section of this chapter, there is derived for linear hyperbolic systems a mathematical equivalent, which is in reality a weak one-sided derivative form (WDF) of the partial differential equations. As previously pointed out, this amounts to obtaining a weak form for the partial differential equation itself, which holds at mesh points even in the presence of a moving discontinuity.

Next, Roe's scheme will be studied in depth, with the motivation of clarifying the distinctions between several closely related methods which derive from his ideas. We will see that Roe's scheme, heuristically derived, can also be obained through utilizing the WDF form of the equations.

\subsection{The WDF Form}

In eqn. $(2.11 b)$, it is assumed that $\bar{U}(x, 0), \frac{\partial \bar{U}}{\partial x}(x, 0)$ have a finite jump discontinuity at $x_{0}$. As is well known, the finite jump discontinuity at $x_{0}$ propagates along the characteristics $x=x_{0}+\lambda_{i} t, i=1,2, \ldots, N$; the solution is otherwise smooth. Attention will be directed to the limiting time derivative 


$$
\bar{U}_{t}^{*}=\lim _{t \rightarrow 0^{+}} \frac{\partial \bar{U}}{\partial t}\left(x_{0}, t\right)
$$

of the solution to the Riemann problem described by eqn. (2.11) .

By employing eqn. (2.18a), the system (2.11a) can be rewritten as

$$
\frac{\partial \bar{U}}{\partial t}+A+\frac{\partial \bar{U}}{\partial X}+A-\frac{\partial \bar{U}}{\partial X}=0
$$

This is called a flux-split form of system (2.11); a well-known form used in numerical analysis [11] to derive finite difference analogs having desirable properties when approximating solution of system (2.11) that have imbedded discontinuities. It is intended to prove:

Theorem 3.1

Let $A(\bar{U})$ be a constant matrix, and let $\bar{U}$ be the solution of the Riemann problem (2.11). Then $\bar{U}_{t}^{*}$ of eqn. (3.1) satisfies the matrix-split equation

$$
\bar{U}_{t}^{*}+A^{-} \frac{\partial \bar{U}_{B}}{\partial x}\left(x_{0}^{+}, 0\right)+A^{+} \frac{\partial \bar{U}_{L}}{\partial x}\left(x_{0}^{-}, 0\right)=0 .
$$




\section{Proof}

Let $v_{i}(x, t)$ be the general component of eqn. (2.12), which satisfies

$$
\frac{\partial V_{i}}{\partial t}+\lambda_{i} \frac{\partial V_{i}}{\partial x}=0, \quad i=1,2, \ldots, N
$$

away from lines $x=x_{0}+\lambda_{i} t, i=1,2, \ldots, N$. since $V_{i}(x, t)$ is a Riemann invariant and the solution of (2.11) is piecewise smooth, $v_{i}(x, t)$ is constant on line $c_{i}: \frac{d x}{d t}=\lambda_{i}, i=1,2, \ldots, N, i . e$.

$$
v_{i}\left(x-\lambda i^{\tau}+\Delta x, t\right)=v_{i}(x+\Delta x, t+\tau)
$$

is true for all $\Delta \mathrm{x}$ and $\tau$. This implies that

$$
\frac{\partial V_{i}}{\partial x}(x, t+\tau)=\frac{\partial V_{i}}{\partial x}\left(x-\lambda_{i} r, t\right),
$$

whenever $\left(x-\lambda_{i}{ }^{r}, t\right)$ is a point where the solution of eqn. (2.11) is smooth. Since the solution is smooth on the lines $\left(x=x_{0}, t>0\right)$ and $\left(t=0, x \neq x_{0}\right)$, it follows that

$$
\lim _{\tau \rightarrow 0^{+}} \frac{\partial V_{i}}{\partial x}\left(x_{0}, \tau\right)=\underset{r \rightarrow 0^{+}}{\lim } \frac{\partial V_{i}}{\partial x}\left(x_{0^{-\lambda}} i^{\tau}, 0\right)
$$


But, from eqn. (3.4)

$\frac{\partial V_{i}}{\partial t}\left(x_{0}, \tau\right)=-\lambda_{i} \frac{\partial V_{i}}{\partial x}\left(x_{0}, \tau\right), \tau>0$.

Using the definition of the limiting time derivative, eqns. (3.8) and (3.7), there results

$\frac{\partial v_{i}^{*}}{\partial t}=-\lambda_{i}\left\{\begin{array}{l}\frac{\partial V_{i}}{\partial x}\left(x_{0}^{+}, 0\right), \lambda_{i}<0 \\ \frac{\partial V_{i}}{\partial x}\left(x_{0}^{-}, 0\right), \lambda_{i}>0\end{array}\right.$

Equation (3.9), together with the piecewise smooth nature of the solution to eqn. (2.11), implies that eqn. (3.3) holds; and therefore, Theorem 3.1 follows.

It is remarked that at points where the weak solution is smooth, eqn. (3.3) collapses back to eqn. (2.11). Thus, eqn. (3.3) is valid generally, at all points of a piecewise smooth solution. Hence, it is a weak one-sided derivative form (WDF) of eqn. (2.1c).

The utility of eqn. (3.3) is that it shows directly how to difference (2.11) in the presence of discontinuities, without fear of differencing across a discontinuity. For example, if first order, one-sided 
differences are used, from eqn. (3.3) there is recovered the scheme

$$
\overline{\mathrm{U}}_{i}^{\mathrm{n}+1}=\overline{\mathrm{U}}_{i}^{\mathrm{n}}-\frac{\Delta t}{\Delta \mathrm{x}}\left(\mathrm{A}^{-} \Delta_{i+1 / 2} \overline{\mathrm{U}}+\mathrm{A}^{+} \Delta_{i-1 / 2} \overline{\mathrm{U}}\right)
$$

Equation $(3.10)$ can also be written as

$$
\overline{\mathrm{U}}_{i}^{\mathrm{n}+1}=\overline{\mathrm{U}}_{i}^{\mathrm{n}}-\frac{\Delta t}{\Delta \mathrm{x}}\left(\Delta \mathrm{F}_{i+1 / 2}^{-}+\Delta \mathrm{F}_{i-1 / 2}^{+}\right)
$$

where

$$
\Delta \mathrm{F}_{i+1 / 2}^{ \pm}=\mathrm{A}^{ \pm} \Delta_{i+1 / 2} \overline{\mathrm{U}}
$$

The fluxes $\Delta F_{i-1 / 2}^{+}$represent information traveling towards $\left(x_{i}, t_{n}\right)$ from the left along right running characteristics; whereas, $\Delta \mathrm{F}_{i+1 / 2}^{-}$represent information moving towards $\left(x_{i}, t_{n}\right)$ from the right along left running characteristics. Thus, the name "upwind biased scheme" is applied to methods where the differencing takes account of the direction of information flow. For the nonlinear case, if $\hat{\mathrm{A}}_{i+1 / 2}$ is a local linearization of $A(\bar{U})$ which holds over an interval $x_{i} \leq x \leq x_{i+1}$, then one can still write a scheme 
corresponding to eqn. (3.10) which is locally

upwind biased, by evaluating the fluxes of eqn. (3.12) in the spatially offset form

$$
\Delta F_{i+1 / 2}^{ \pm}=\hat{A}_{i+1 / 2}^{ \pm} \Delta_{i+1 / 2} \bar{U}
$$

It will be shown in the sequel that the upwind biased scheme of eqn. (3.13), derived here using the WDF form of eqn. (2.1c) evidenced by eqn. (3.3), reduces to a certain scheme of Roe $[9,10]$.

\subsection{Roe's Scheme}

Roe's approximation to the nonlinear Riemann problem [10] involves at each cell interface the exact solution of a locally linearized system

$$
\begin{aligned}
& \frac{\partial \bar{U}}{\partial t}+\tilde{A} \frac{\partial \bar{U}}{\partial X}=0 \\
& \bar{U}(x, 0)= \begin{cases}\bar{U}_{L}, & x<0 \\
\bar{U}_{R,} & x>0\end{cases}
\end{aligned}
$$

Here $\tilde{\mathrm{A}}\left(\overline{\mathrm{U}}_{\mathrm{L}}, \overline{\mathrm{U}}_{\mathrm{R}}\right)$ is the mean value Jacobian matrix, which is piecewise constant and satisfies the property (W): 
(W)

$$
\tilde{\mathrm{A}}\left(\overline{\mathrm{U}}_{L}, \overline{\mathrm{U}}_{\mathrm{R}}\right) \times\left(\overline{\mathrm{U}}_{\mathrm{R}}-\overline{\mathrm{U}}_{L}\right)=\mathrm{F}_{\mathrm{R}}-\mathrm{F}_{\mathrm{L}}
$$

where

$$
\overline{\mathrm{U}}_{\mathrm{L}}=\overline{\mathrm{U}}_{\mathrm{i}}^{\mathrm{n}} \text { and } \quad \overline{\mathrm{U}}_{\mathrm{R}}=\overline{\mathrm{U}}_{\mathrm{i}+\mathrm{I}}^{\mathrm{n}} \text {. }
$$

\section{View 1: A First Approximate Riemann Solver}

In the first paper [9], Roe suggests using

approximate solutions to the Riemann problem to simplify Godunov's scheme. Thus, the modified scheme (2.19)

becomes

$$
\overline{\mathrm{U}}_{i}^{n+1}=\overline{\mathrm{U}}_{i}^{\mathrm{n}}-\frac{\Delta t}{\Delta \mathrm{x}}\left[F_{i+1 / 2}^{*}-F_{i-1 / 2}^{*}\right]
$$

where $F_{i+1 / 2}^{*}=F\left(U_{i+1 / 2}^{*}\right)$ is the numerical flux emerging from the approximate problem (3.14).

Thus, for the linear case, Roe's scheme $(3.16)$ is exactly the same as Godunov's scheme (2.19); hence, also equivalent to the flux splitting (2.36).

We now concentrate on a further scheme which Roe $[9,10]$ developed for nonlinear hyperbolic systems (2.1). As a result, we are able to consider another two views which emerge from Roe's work, one of which Roe did not relate in his original papers $[9,10]$. 
View 2: Passage of Information By Eigenvector Projection Fixing attention on the interval $x_{i} \leq x \leq x_{i+1}$ and states $\bar{U}_{I}=\bar{U}_{i}^{n}, \bar{U}_{R}=\bar{U}_{i+1}^{n}$, let $\bar{U}_{i+1 / 2}=V\left(\bar{U}_{I}, \bar{U}_{R}\right)$ be some symmetric average of the given data. Then system (2.11) can be locally linearlized using the mean value Jacobian matrix $\tilde{\mathrm{A}}=\mathrm{A}\left(\overline{\mathrm{U}}_{\mathrm{i}+1 / 2}\right)$. Roe [9] questions how the data increment $\bar{U}_{R}-\bar{U}_{I}=\Delta_{i+1 / 2} \bar{U}$ should be used in construction of numerical algorithms. He first chooses $V\left(\bar{U}_{L}, \bar{U}_{R}\right)$ in a specific manner $[10]$, such that the mean value Jacobian matrix $\tilde{A}=A(V)$ satisfies the property

$$
\tilde{A}\left(\bar{U}_{I}, \bar{U}_{R}\right) \times\left(\bar{U}_{R}-\bar{U}_{I}\right)=F_{R}-F_{L} \cdot
$$

Now, suppose the data increment is projected onto the right eigenvectors $R_{i+1 / 2}^{\mathrm{k}}$ of $\tilde{\mathrm{A}}_{i+1 / 2}$ :

$$
\Delta_{i+1 / 2} \bar{U}=\sum_{k=1}^{N} \alpha_{i+1 / 2}^{k} R_{i+1 / 2}^{k}
$$

Then, solely because of property $(w)$, the quantity

$$
\tilde{\mathrm{A}}_{i+1 / 2} \Delta_{i+1 / 2} \overline{\mathrm{U}}=\sum_{\mathrm{k}=1}^{\mathrm{N}} \alpha_{i+1 / 2}^{\mathrm{k}} \lambda_{i+1 / 2}^{\mathrm{k}} \mathrm{R}_{i+1 / 2}^{\mathrm{k}}
$$


is actually a physical flux increment,

$$
F_{i+1}-F_{i}=\Delta F_{i+1 / 2}=\Delta F_{i+1 / 2}^{+}+\Delta F_{i+1 / 2}^{-}
$$

where

$$
\Delta F_{i+1 / 2}^{+}=\sum_{k=m+1}^{N} \alpha_{i+1 / 2}^{k} \lambda_{i+1 / 2}^{k} R_{i+1 / 2}^{k}
$$

and

$$
\Delta F_{i+1 / 2}^{-}=\sum_{k=1}^{m} \alpha_{i+1 / 2}^{k} \lambda_{i+1 / 2}^{k} R_{i+1 / 2}^{k}
$$

Here, $\Delta F^{+}$represents information conveyed to the right on characteristics whose corresponding wave speeds ${ }_{\lambda} \mathrm{k}, \mathrm{k}=\mathrm{m}+1, \mathrm{~m}+2, \ldots, \mathrm{N}$ are positive; whereas, $\Delta \mathrm{F}^{-}$represents information traveling to the left on characteristis whose corresponding wave speeds $\lambda k, k=1,2, \ldots, m$ are negative.

Roe's second use of the linearizing matrix, $\tilde{\mathrm{A}}$, is, thus, synthesis of the numerical scheme

$$
\overline{\mathrm{U}}_{i}^{\mathrm{n}+1}=\overline{\mathrm{U}}_{i}^{\mathrm{n}}-\frac{\Delta t}{\Delta \mathrm{x}}\left[\Delta \mathrm{F}_{i-1 / 2}^{+}+\Delta \mathrm{F}_{i+1 / 2}^{-}\right]
$$


From a heuristic standpoint, eqn. (3.21) represents a flux splitting method. To allow proper signal flow by left and right moving waves requires the spatially offset flux evaluations evidenced by $(3.21)$.

\section{VIEW 3: A Second Approximate Riemann Solver}

We now obtain, independently of Roe, a second approximate Riemann solver. However, in this approach we still use the concept of a locally linearlized matrix, $\hat{A}$. Note that, whether or not $\hat{A}$ is Roe's mean value Jacobian matrix, one can ask for a vector $F_{i+1 / 2}^{*}$ which satisfies the equations

$$
\begin{aligned}
& F_{i+1 / 2}^{*}-F_{i}=\sum_{k=1}^{m} \alpha_{i+1 / 2}^{k} \lambda_{i+1 / 2}^{k} R_{i+1 / 2}^{k}=\Delta F^{-} \\
& F_{i+1}-F_{i+1 / 2}^{*}=\sum_{k=m+1}^{N} \alpha_{i+1 / 2}^{k}{ }_{i+1 / 2}^{k} R_{i+1 / 2}^{k}=\Delta F^{+} .
\end{aligned}
$$

The solution, of course, is

$$
F_{i+1 / 2}^{*}=\frac{F_{i}+F_{i+1}}{2}-\frac{\left|\hat{A}_{j+1 / 2}\right|}{2} \Delta_{i+1 / 2} \bar{U}
$$


Here $\hat{\mathrm{A}}_{i+1 / 2}=\hat{\mathrm{A}}\left(\overline{\mathrm{U}}_{i+1 / 2}\right)=\hat{\mathrm{A}}\left(\mathrm{V}\left(\overline{\mathrm{U}}_{i}, \overline{\mathrm{U}}_{i+1}\right)\right)$, but $\mathrm{V}$ is not necessarily as chosen by Roe [10]. It is remarked, there may or may not exist a vector $U^{*}$ such that $F_{i+1 / 2}^{*}=F\left(U^{*}\right)$; hence, $F_{i+1 / 2}^{*}$ is strictly a hypothetical flux vector. Also, eqn. (3.22c) may or may not represent a better approximate Riemann solver than either of Roe's choices.

VIEW 1: $\quad F\left(U_{i+1 / 2}^{*}\right)$, or

VIEW 2: $\quad F_{i+1 / 2}^{*}=\frac{F_{i}+F_{i+1}}{2}-\frac{\left|\tilde{\mathrm{A}}_{i+1 / 2}\right|}{2} \Delta_{i+1 / 2} \overline{\mathrm{J}}$.

However, it will be useful in establishing the following theorem:

\section{Theorem 3.2:}

When $\hat{A}_{i+1 / 2}=\tilde{A}_{i+1 / 2}$ is characterized by Roe's property (W) the numerical scheme of the form (2.19) which employs the approximate Riemann solver of eqn. (3.22c) faithfully generalizes the flux splitting property possessed by Godunov's scheme in the linear case. 


\section{Proof}

By substituting the flux vectors of eqn.

(3.22c) at interfaces $x_{i+1 / 2}$ and $x_{i-1 / 2}$ into

eqn. (3.16), there results:

$$
\bar{U}_{i}^{n+1}=\bar{U}_{i}^{n}-\frac{\Delta t}{\Delta x}\left\{\left[\frac{F_{i}+F_{i+1}}{2}-\frac{\left|\tilde{A}_{i+1 / 2}\right|}{2} \Delta_{i+1 / 2} \bar{U}\right]\right.
$$

$$
\left.-\left[\frac{F_{i-1}+F_{i}}{2}-\frac{\left|\tilde{A}_{i-1 / 2}\right|}{2} \Delta_{i-1 / 2} \bar{U}\right]\right\}
$$

Since $\tilde{\mathbb{A}}_{i+1 / 2}, \tilde{A}_{i-1 / 2}$ possess the property (W) of Roe, eqn. (3.25) can be simplified as follows:

$$
\begin{aligned}
\overline{\mathrm{U}}_{i}^{\mathrm{n}+1}=\overline{\mathrm{U}}_{i}^{\mathrm{n}}-\frac{\Delta t}{\Delta \mathrm{x}} & {\left[\frac{\tilde{\mathrm{A}}_{i+1 / 2}}{2} \Delta_{i+1 / 2} \overline{\mathrm{U}}-\frac{\left|\tilde{\mathrm{A}}_{i+1 / 2}\right|}{2} \Delta_{i+1 / 2} \overline{\mathrm{U}}\right.} \\
& \left.+\frac{\tilde{\mathrm{A}}_{i-1 / 2}}{2} \Delta_{i-1 / 2} \overline{\mathrm{U}}+\frac{\left|\tilde{\mathrm{A}}_{i-1 / 2}\right|}{2} \Delta_{i-1 / 2} \overline{\mathrm{U}}\right]
\end{aligned}
$$

By eqn. (2.18), eqn. (3.26) becomes 


$$
\overline{\mathrm{U}}_{i}^{\mathrm{n}+1}=\overline{\mathrm{U}}_{i}^{\mathrm{n}}-\frac{\Delta t}{\Delta \mathrm{x}}\left[\tilde{\mathrm{A}}_{i+1 / 2}^{-} \Delta_{i+1 / 2} \overline{\mathrm{U}}+\tilde{\mathrm{A}}_{i-1 / 2}^{+} \Delta_{i-1 / 2} \overline{\mathrm{U}}\right]
$$

This completes the proof.

Actually, the local WDF form also indicates that eqn. (3.27) is the proper first order upwind scheme to choose as a generalization. Further supporting evidence for this choice is now provided.

Note, as previously stated, that eqn. (3.22) affords an approximate flux solution for the nonlinear Riemann problem. Moreover, the numerical flux difference applied in (3.16) becomes

$$
\begin{aligned}
& \Delta F^{*}=F_{i+1 / 2}^{*}-F_{i-1 / 2}^{*} \\
& =\hat{A}_{i+1 / 2}^{-} \Delta_{i+1 / 2} \bar{U}+\hat{A}_{i-1 / 2}^{+} \Delta_{i-1 / 2} \bar{U}+I_{i+1 / 2}+I_{i-1 / 2}
\end{aligned}
$$

where $\quad L_{i+1 / 2}=\frac{F_{i+1}-F_{i}-\hat{A}_{i+1 / 2} \Delta_{i+1 / 2} \bar{U}}{2}$

For eqn. (3.28a) to be the incremental numerical flux of an upwind biased first order accurate method, it is 
necessary that $L_{i+1 / 2}=0\left(\Delta^{2}\right)$; where $\Delta$ is the discretization parameter. Therefore, there is now sought the matrix $\hat{A}_{i+1 / 2}$ which locally solves on each interval $x_{i} \leq x \leq x_{i+1}$ the following minimization problem:

Choose $\hat{A}_{i+1 / 2}$ to make the $I_{i+1 / 2}$ as small as possible, i.e. the $\hat{A}_{i+1 / 2}$ so chosen is the solution of

$\min _{\lambda{ }_{i+1 / 2}^{k} \in S}\left\|L_{i+1 / 2}\left(\bar{U}_{i}^{n}, \bar{U}_{i}^{n+1} ; \lambda_{i+1 / 2}^{k}, k=1,2, \ldots, N\right)\right\|$

in any norm, with $\lambda$ running over the eigenvalues of $\hat{A}_{i+1 / 2}$, and $S$ the $\operatorname{set}\left\{\lambda{ }_{i+1 / 2}^{k}\right\}_{k=1}^{N}$ which approximates the local wave speeds of eqn. (2.1).

\section{Remark 1.}

Such a choice gives a scheme which in the nonlinear case should closely approximate the correct passage of information flow indicated by the WDF differencing of eqn. (3.3) . 
Remark 2.

The global minimum of (3.29) has value zero, achieved

when $\hat{A}_{i+1 / 2}$ has the proeprty (W), (i.e. $\hat{A}_{i+1 / 2}=\tilde{A}_{i+1 / 2}$ ). In this case, the incremental fluxes from eqns. (3.28a) and $(3.24)$ are identical.

Remark 3.

For the Euler equations of ideal compressible flow in one dimension, Roe $[9,10]$ derives explicitly the vector $\bar{U}_{i+1 / 2}$ which pinpoints the global minimum value of zero for problem (3.29). The upwind scheme of Roe coincides precisely with our upwind scheme obtained from the WDF form, eqns. (3.10-3.13).

Remark 4.

When the linearizing matrix does not possess the property $(W)$, use of the numerical fluxes obtained from eqn. (3.24) leads to a class of numerical methods not studied by Roe. In some physical applications where very strong shocks arise, this form is thought to be superior [4]. 


\section{Chapter 4}

A MOVING BOUNDARY PROBLEM OF TRANSITIONAL BALLISTICS

Acquisition of detailed knowledge concerning the internal and external ballistics involved in the firing of gun weapons is a major goal of military and industrial research. The design of silencers, as well as improved accuracy of fire and efficient design of anti-recoil devices, cannot be achieved without elaborate analyses of the flow field in the chamber and near field muzzle region of a gun, in the short interval of time between the initial firing of the projectile and its exit through the precursor shock wave. Experimental techniques have evolved to a high degree of sophistication, so that a complete descripton of one firing can be obtained by a combination of measurements, photographs and data processing. Innovative design, however, requires similar analysis for a large number of cases, using different models; this task may be too time consuming and expensive to be accomplished by experimental means. Numerical analysis offers a possible alternative approach. Numerical analyses of the transitional ballistics problem has proceeded in stages of incresingly complex modelling of the physical problem. Moretti [1] has studies the effects of the precursor flow. Estimates of blast wave overpressure can be obtained from numerical 
calculation of flow from the open end of shock tube [2]. More realistic simulations which include the bullet and muzzle brake in the weapons model, for both two and three dimensional flow, have been performed as well [3].

Two different approaches to simulation of the projectile motion have emerged: Widhopf and Buell [3] have used the Godunov method (away from the bullet) together with a physically motivated alagorithm for treating expanding and compressing cells in back and front of the bullet. The sequence of operations proposed in [3] has been modified by Fansler [5], in the interest of getting rid of a non-physical pressure build up on the back of the projectile. Carofano [4] employed the second-order Harten shock capturing algorithm for calculation at cells away from the bullet, and an approach which employs the method of characteristics and Riemann invariants for updating cells adjacent to the bullet. since no rigorous assessments have been made concerning the merits of algorithms for projectile movement during flow simulation, one purpose of this study is to provide such assessment, for a new update algorithm which we shall obtain. By treating the expanding cells through a finite volume technique, mathematical relations for updating flow in the expanding cell are obtained. Analysis of these equations shows clearly why the heuristic Fansler modification to Widhopf's approach is necessary. However, in 
computational fluid dynamics the final test of a mathematical model is a comparison of results, for a case in which, if possible, the exact solution is known or else can be experimentally obtained. In the present instance we have chosen the moving boundary problem provided by a constant velocity piston, impulsively started and witharawing from a gas initially at rest. The solution, of course, is the first half of the well-known shock tube problem of Riemann [18], starting with the gas at rest and extending to the moving contact surface. The analytic solution is well-known, and the indicated portion back of the contact surface provides a natural standard of comparison for the projectile movement algorithm.

\subsection{A One-Dimensional Model}

In general, calculation of bullet motion would be performed in an axisymmetric coordinate system (see Fig. 4.1). However, for ease of illustration, in this section a one-dimensional Cartesian model for the moving piston problem will be considered. As the theoretical solution to such a problem is well known, comparison with numerical results can be used to validate the algorithm developed. 


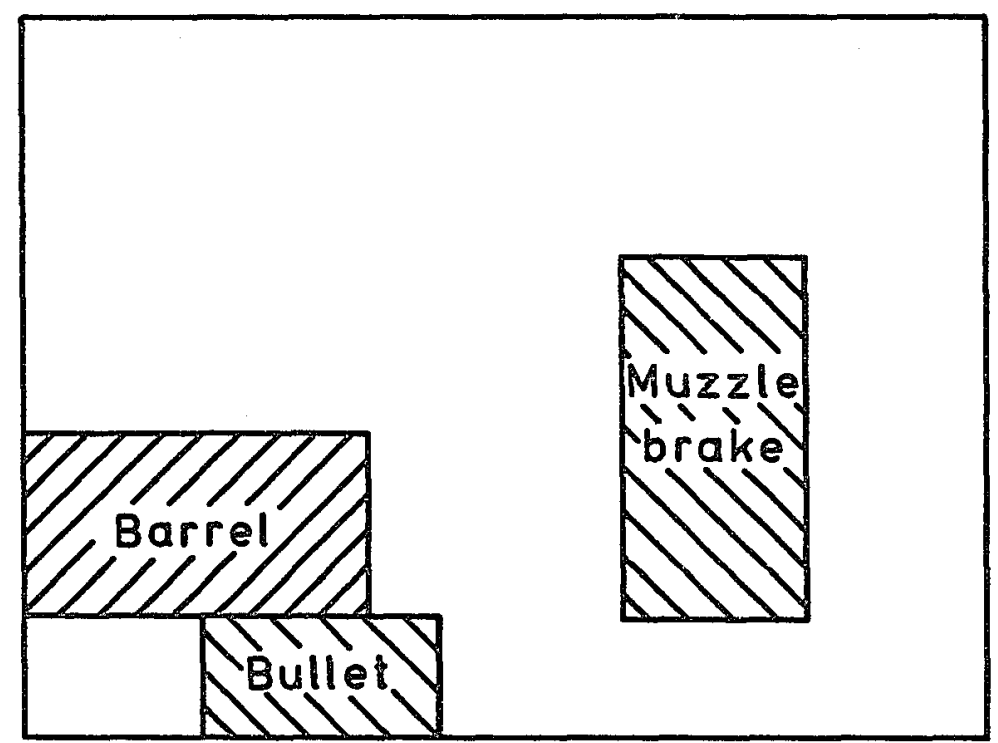

Fig. 4.1 Typical Weapons Configuration Axisymmetric Geometry. 


\subsubsection{Algorithm Overview}

We shall make certain simplifying assumptions:

(i) The bullet has square ends, and moves at constant speed $u_{B}$.

(ii) Roe's adaption of the Godunov method will be used for cells away from the piston. The expanding cell algorithm developed here will be used at the moving pistion (see Fig. 4.2).

As the bullet moves through the computational domain, the cell in back of the bullet may be viewed as expanding in volume, while the cell in front of the bullet is compressing. Initially we shall be concerned with cells behind the bullet.

As the back of the bullet passes a cell interface, the cell is allowed to expand, creating an irregular size cell. This cell is allowed to grow until it is at least two cells long, then split, and the process is continually repeated (see Fig. 4.3). Of course, by considering the CFL stability condition, criteria can be developed for splitting the expanding cell when its length is less than two cells, which improves the accuracy of the computation. 


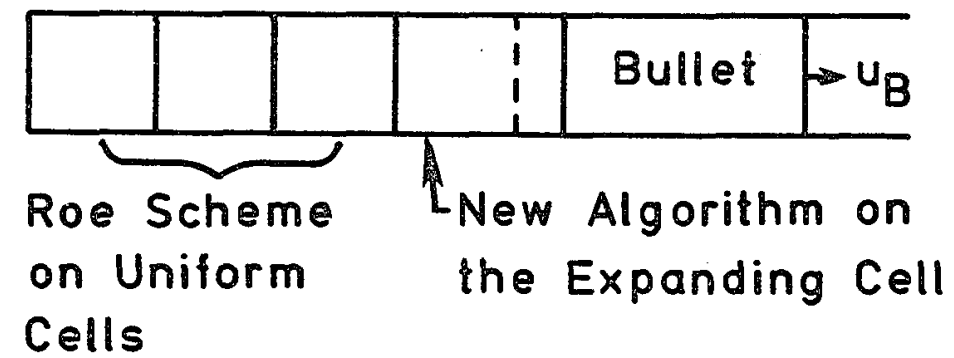

Fig. 4.2 Algorithm Schematic 

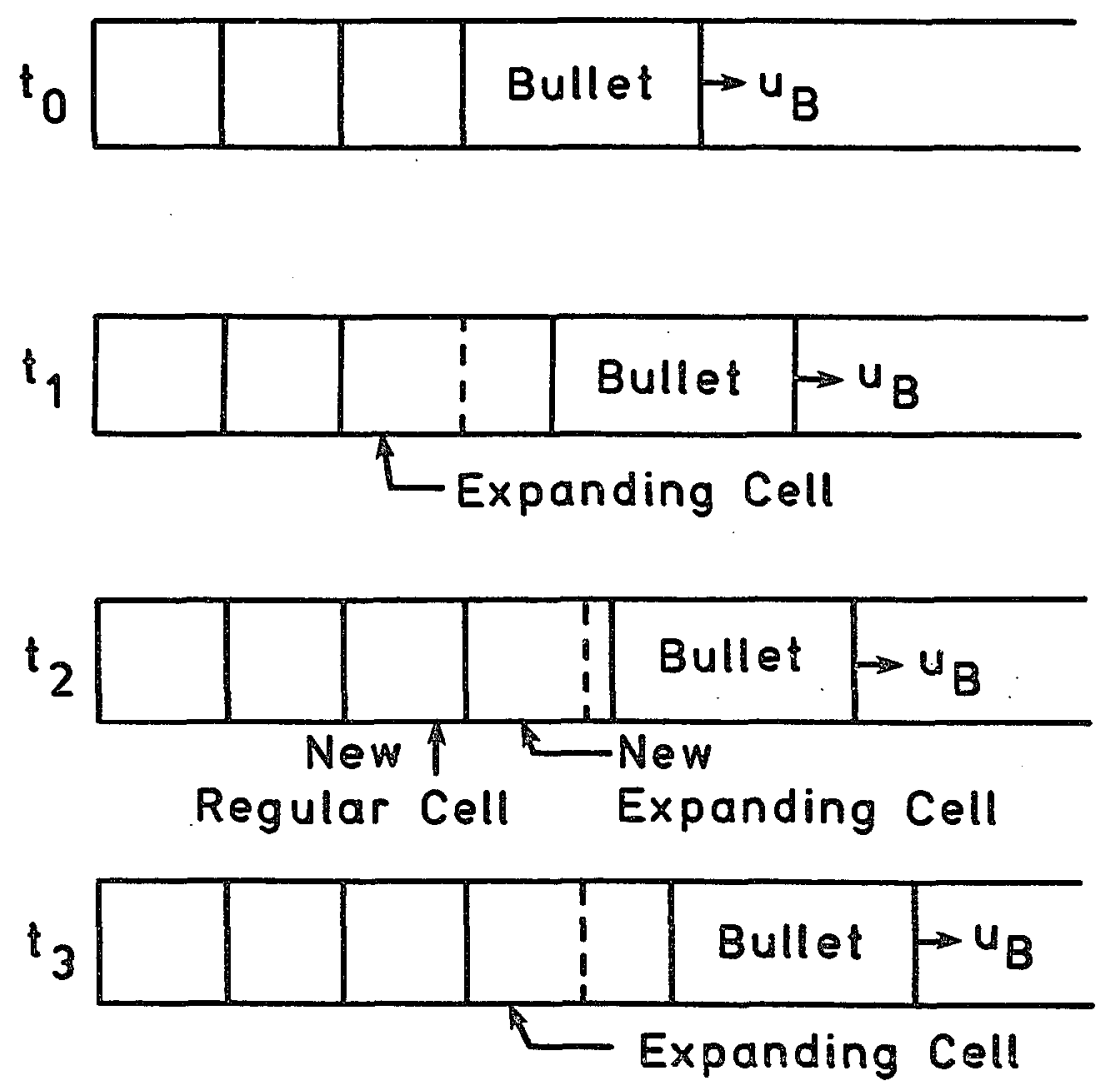

Fig.4.3 Cell Division 
4.1.2 Governing Equations of Idealized Compressible Flow The conservation equations for one dimensional, ideal compressible flow may be written as [3]

$$
\frac{a \bar{U}}{\partial t}+\frac{a F(\bar{U})}{\partial X}=0
$$

where

$$
\bar{U}=\left[\begin{array}{l}
\rho \\
\rho u \\
\rho E
\end{array}\right], \quad F(\bar{U})=\left[\begin{array}{l}
\rho u \\
P+\rho u^{2} \\
(P+\rho E) u
\end{array}\right]
$$

and

$$
\begin{aligned}
& \mathrm{x}=\text { space coordiante } \\
& \mathrm{t}=\text { time } \\
& \mathrm{u}=\text { particle velocity } \\
& \rho=\text { density } \\
& \mathrm{E}=\text { specific total energy }=e+\frac{\mathrm{u}^{2}}{2} \\
& \mathrm{e}=\text { specific interval energy }=c_{\mathrm{v}} \mathrm{T} \\
& \mathrm{c}_{\mathrm{v}}=\text { specific heat at constant volume } \\
& \mathrm{T}=\text { temperature } \\
& \mathrm{P}=\text { pressure }
\end{aligned}
$$

These equations will be used in deriving a scheme which accounts for the physics of cell volume changes as the 
projectile moves.

\subsubsection{A Local Finite Volume scheme}

Integrating eqn. (4.1a) over the cell exhibited in

Fig. 4.4 yields, through Green's Theorem for the plane,

$$
\int_{\Gamma} \overline{\mathrm{U} d x}=\int_{\Gamma} F(\overline{\mathrm{U}}) d t
$$

Consider the mean value relations

$$
\Delta x \bar{U}_{i}^{n}=\int_{x_{i-1 / 2}}^{x_{i+1 / 2}} \bar{U}\left(x, t_{n}\right) d t
$$

and

$$
(\Delta x+\epsilon) \bar{U}_{i}^{n+1}=\int_{x_{i-1 / 2}}^{x_{i+1 / 2}+\epsilon} \bar{U}\left(x, t_{n+1}\right) d x
$$

By use of these mean value relations, eqn. (4.2) becomes

$$
\overline{\mathrm{U}}_{i}^{\mathrm{n}+1}=\frac{1}{\Delta \mathrm{x}+\epsilon}\left[\overline{\mathrm{U}}_{i}^{\mathrm{n}} \Delta \mathrm{x}-\int_{\Gamma} F(\overline{\mathrm{U}}) \mathrm{dt}+\int_{\text {Wall }} \overline{\mathrm{U}} \mathrm{dx}\right]
$$

or 


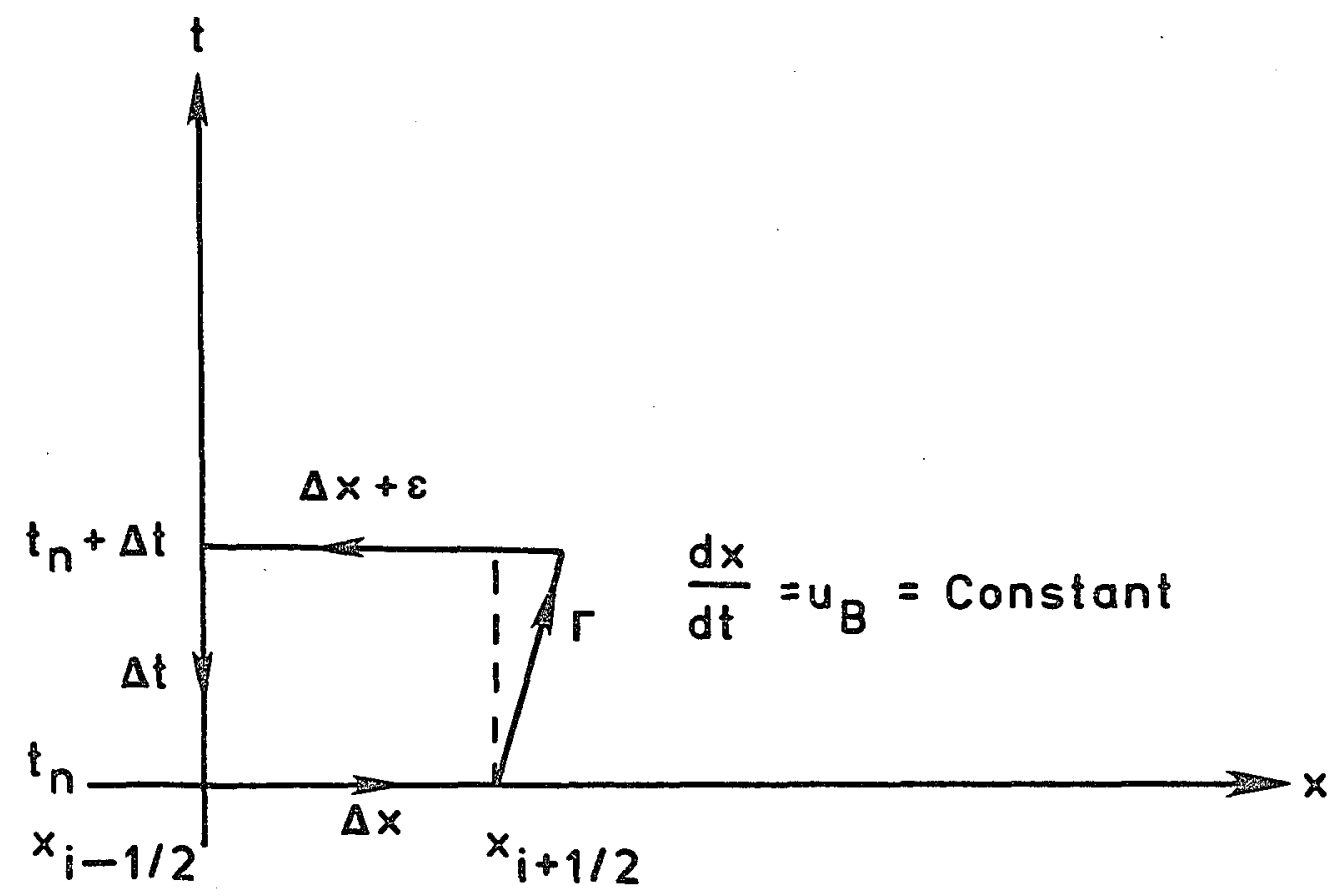

Fig.4.4 Phase Plane 


$$
\begin{aligned}
\overline{\mathrm{U}}_{i}^{\mathrm{n}+1} & =\frac{1}{\Delta \mathrm{x}+\epsilon}\left[\overline{\mathrm{U}}_{i}^{\mathrm{n}} \Delta \mathrm{x}-\int_{\text {wall }} F(\overline{\mathrm{U}}) d t\right. \\
& \left.+\int_{t_{n}}^{t_{n+1}} F\left(\overline{\mathrm{U}}\left(\mathrm{x}_{i-1 / 2}, t\right)\right) d t+\int_{\text {walI }} \overline{\mathrm{U}} \mathrm{dx}\right]
\end{aligned}
$$

The first integral can be evaluated (second-order accurate) using the trapezoidal scheme; whereas, the Riemann problem of the Godunov method [8] provides a means for dealing with the second. The result is:

$\overline{\mathrm{U}}_{i}^{n+1}=\frac{\overline{\mathrm{U}}_{i}^{\mathrm{n}} \Delta \mathrm{x}-\left[\left(\mathrm{F}^{\mathrm{W}}+\mathrm{F}_{\mathrm{W}}\right) / 2-\mathrm{u}_{B}\left(\overline{\mathrm{U}}^{\mathrm{W}}+\overline{\mathrm{U}}_{\mathrm{W}}\right) / 2-\mathrm{F}_{i-1 / 2}^{*}\right] \Delta t}{\Delta \mathrm{x}+\epsilon}$

where the "w" subscript or superscript denotes values on the back surface of the moving piston, at the current time level and the advanced time level, respectively. Here $(*)_{i-1 / 2}$ represents solving a local Riemann problem on interface $x_{i-1 / 2}$.

Interpretation of Equation (4.6):

We note that the flux expression 


$$
F=\left[\begin{array}{l}
\rho u \\
P+\rho u^{2} \\
(P+\rho E) u
\end{array}\right]
$$

contains transport terms, whereas the boundary condition at a moving wall prohibits transport across the wall. Hence, the presence of the second term in eqn. (4.6) is necessary simply to assure no transport occurs across the moving wall; for momentum, density, or specific total energy. By defining a corrected wall flux as

$$
F_{W}^{C}=\left[\begin{array}{l}
0 \\
P_{W} \\
P_{W} u_{B}
\end{array}\right]
$$

we see that eqn. $(4.6)$ becomes

$$
\bar{U}_{i}^{n+1}=\frac{\bar{U}_{i}^{n} \Delta x-\left[\left(F_{C}^{W}+F_{w}^{C}\right) / 2-F_{i-1 / 2}^{*}\right] \Delta t}{\Delta x+\epsilon}
$$

Here a raised subscript on $F_{C}^{W}$, for $w$, denotes advanced time level. The terms in the flux of eqn. (4.7) represent pressure forces and pressure work occurring due to the 
moving wall. In eqn. $(4.8), F^{*}$ is a flux calculated at non-moving interfaces by solving the usual Riemann problem. The algorithm of eqn. (4.8), together with some compatible choice of shock capturing algorithm for updating fixed cells not adjacent to the moving projectile, provides an aesthetically pleasing mathematical approach to inclusion of the moving projectile in blast wave simulations. The counterpart of eqn. (4.8) for a compressing cell (projectile front) requires replacement of $\epsilon$ by $-\epsilon$ and $\Delta t$ by $-\Delta t$ in eqn. $(4.8)$, with $F^{*}$ evaluated now at $x_{i+1 / 2}$.

\subsubsection{Axisymmetric Problems}

For two dimensional or axisymmetric flow, the method of operator splitting allows eqn. (4.8) to be applied on the $x$-sweep, with no expanding cell to be concerned about on the $y$-sweep, for flat-fronted projectiles. According to Moretti [1], the assumption of a flat-fronted projectile is no real restriction, as the fluid next to the bullet assumes, generally, a rigid body type motion. However, cells at or near the corners of the bullet require special handling. The treatment here reverts to the use of Godunov's method.

4.2 Numerical Validation of the Expanding Cell Method Consider a shock tube with initial values $P_{2}=1.0$, $o_{2}=1.0, u_{2}=0 . ; P_{1}=0.1, \rho_{1}=0.125, u_{1}=0$. If $a$ 
piston is impulsively started from rest and moves at the constant speed of the contact surface for the shock tube, with $P_{2}, \rho_{2}, u_{2}$ the initial state behind the piston, the solutions of the piston and shock tube problem are theoretically identical, on their common domain. Figures 4.5-4.7 show a comparison of numerical results for the two problems. Roe's method is used globally for the shock tube problem; otherwise away from the expanding cell. The pressure comparison of Fig. 4.5 shows rather small percent differences. Except near the contact surface, the results for density, exhibited in Fig. 4.6, are also good. The contact surface is badly smeared, for the shock tube calculation; hence, differences nearby are expected to be large, as the standard of comparison alters. Larger percent differences, on the order of a few percent, are seen in the velocity comparison of Fig. 4.7. This may be accounted for partly by the fact that the piston speed is fixed; whereas, for the shock tube the contact surface speed is influenced by how well the shock speed is resolved. Therefore, the contact surface speed is subject to aggregate numerical error, which leads to a poor comparison, in the plateau region of Fig. 4.7. However, a more likely explanation is the influence of the numerical boundary condition used at the piston, which consists of computing wall pressure (hence, wall flux) on the assumption that either a compression or expansion wave is between the last grid point and the 


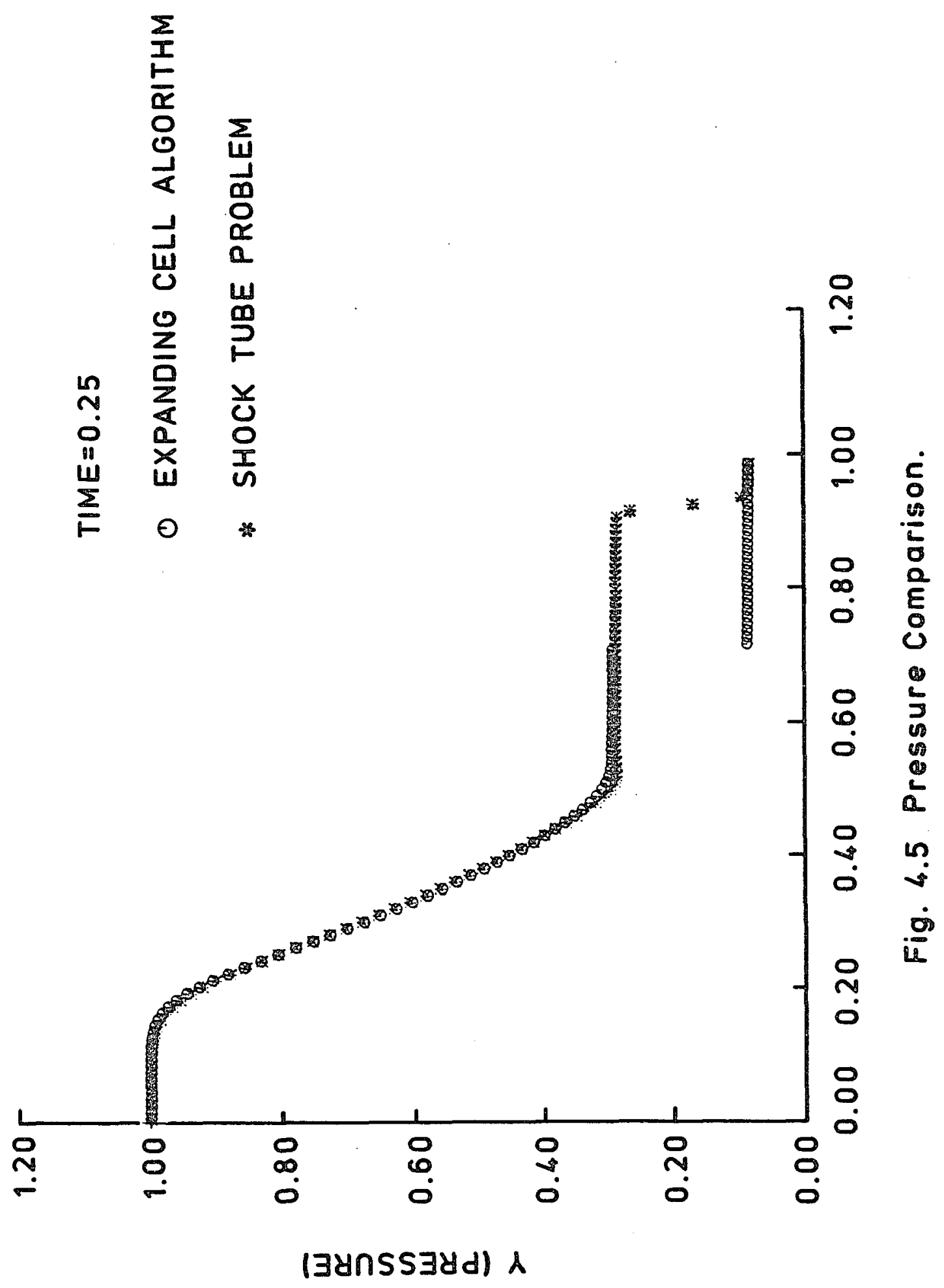




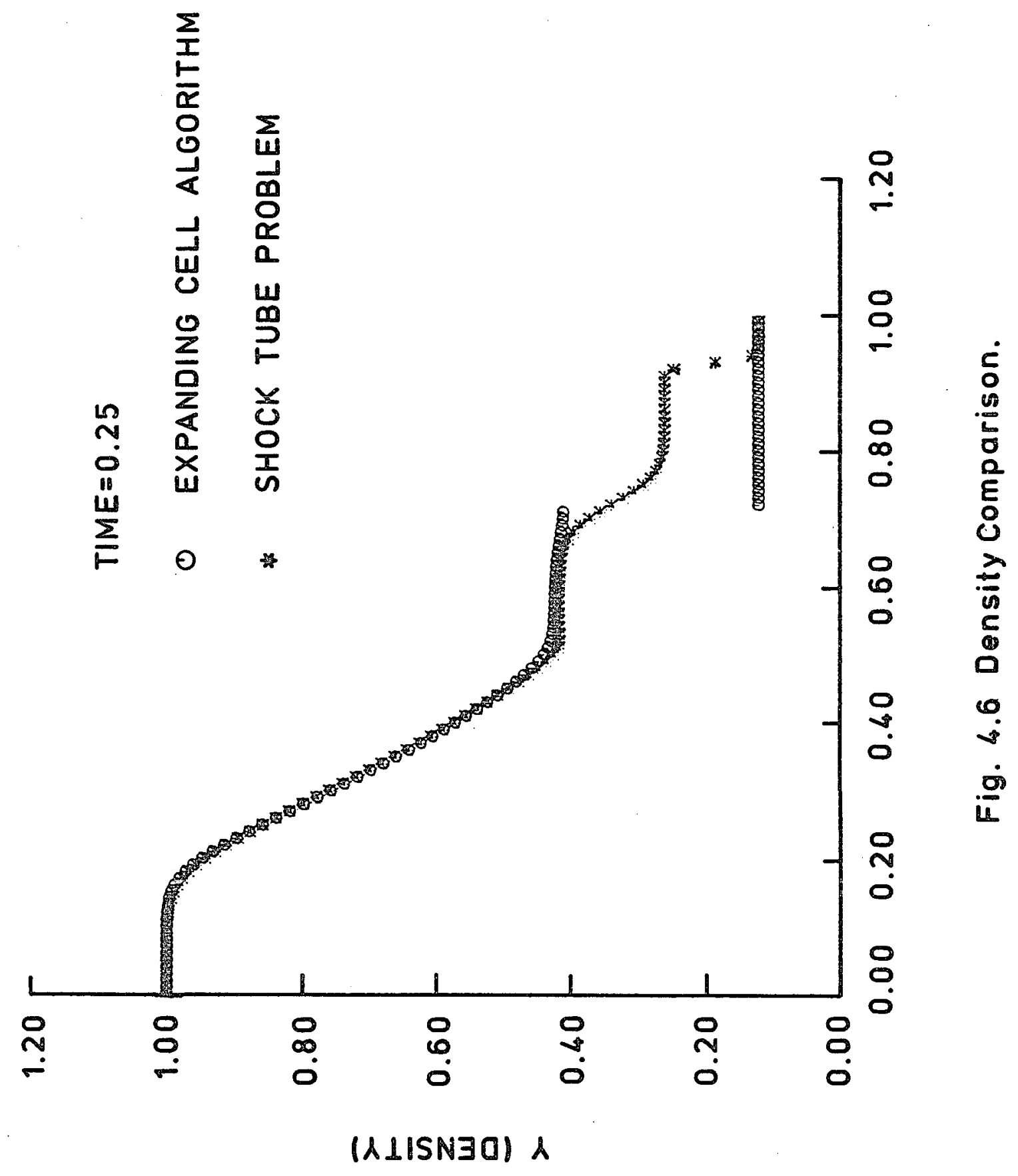




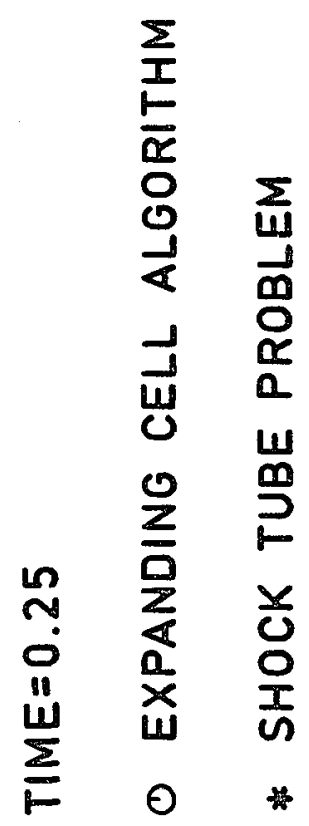

동

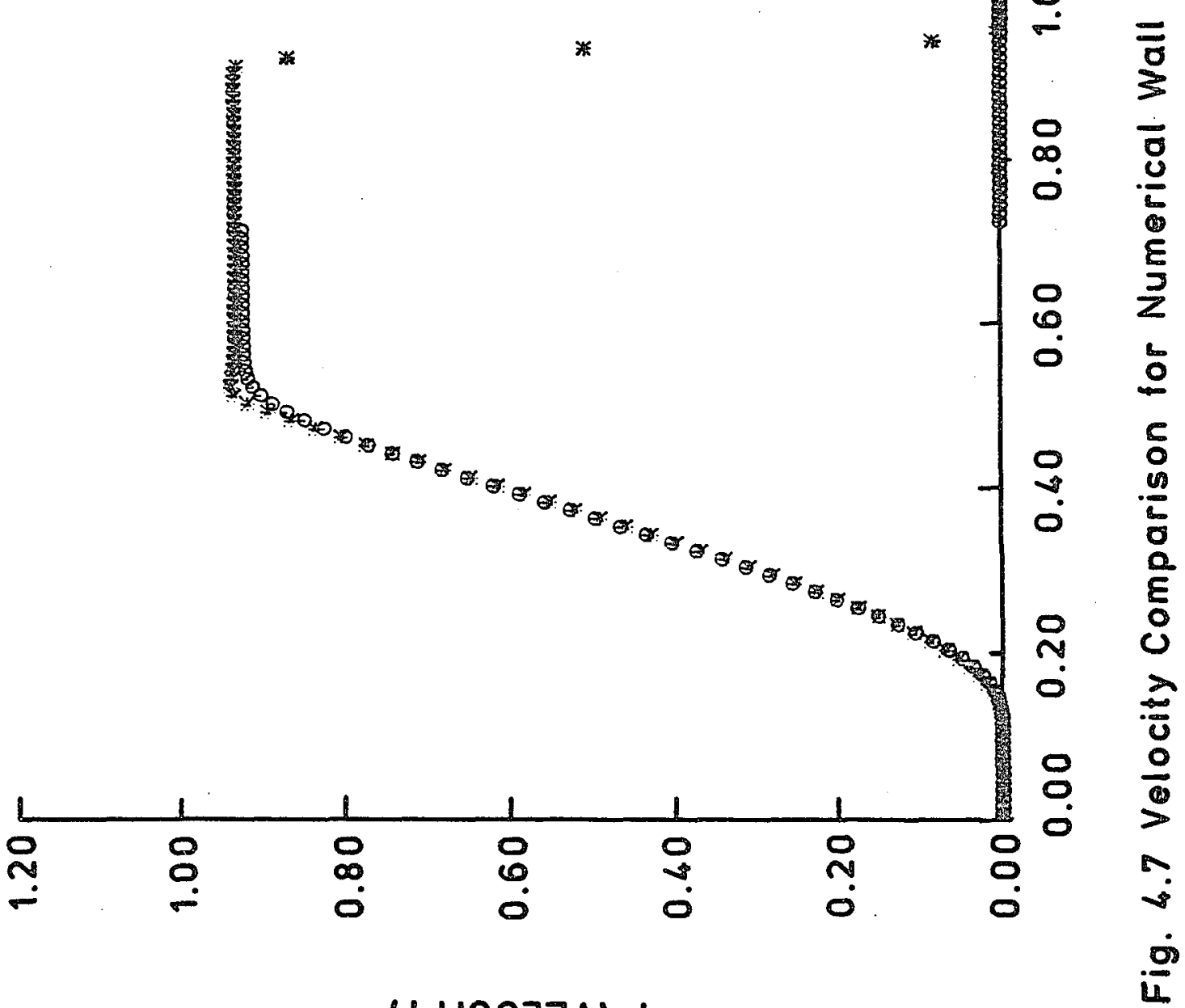

(A1) 
piston (see Chap. 6). Figure 4.8 shows the improvement which is a result of using the correct wall pressure. It should be noted that, in general, such a correct pressure value is not available; hence, the rumerical boundary treatment. 


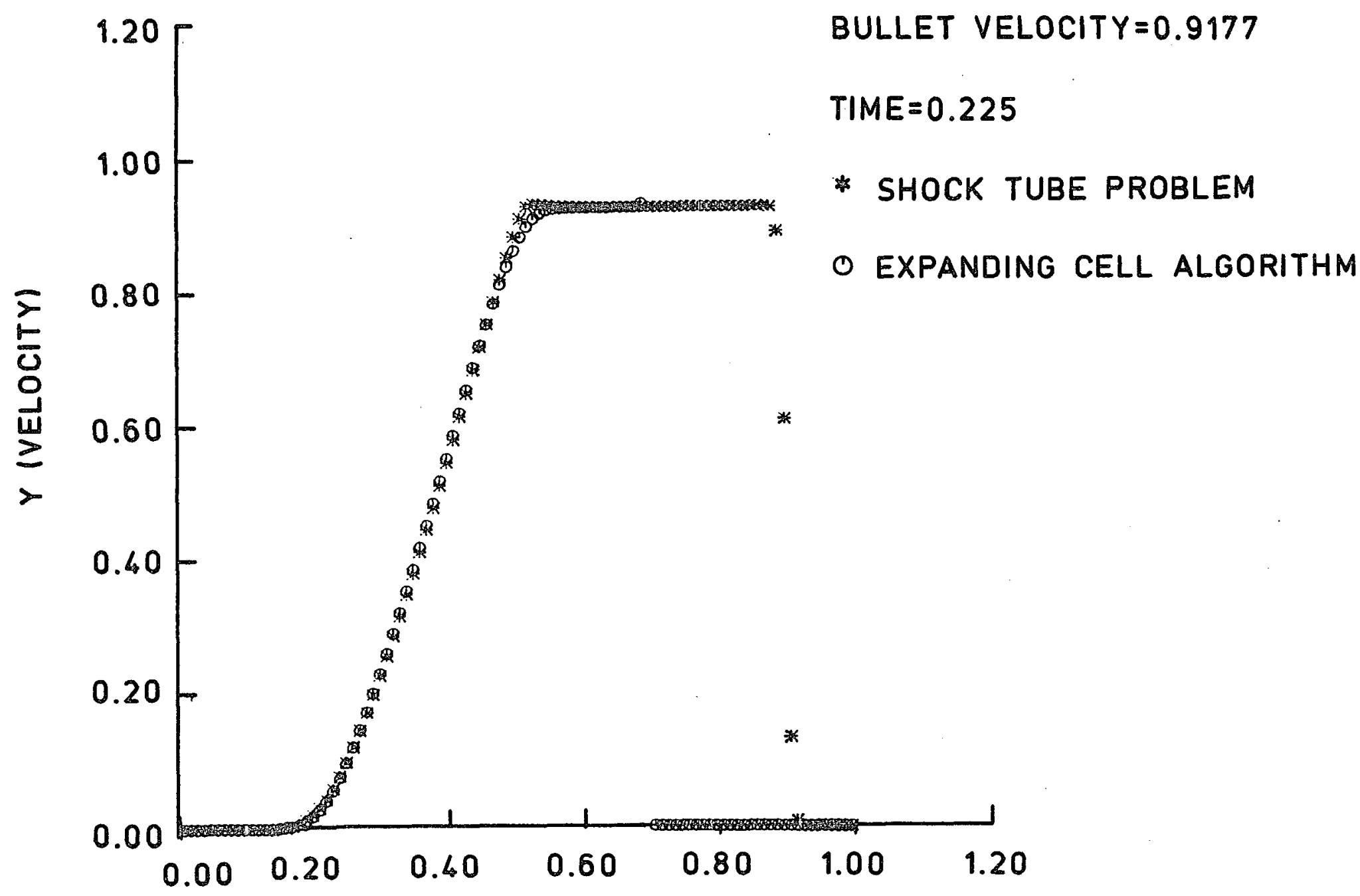

Fig. 4.8 Velocity Comparison: Theoretical Wall Boundary Condition 


\section{Chapter 5}

\section{SUPERSONIC PROJECTILE MOTION}

In this chapter we study the idealized compressible flow around a projectile which moves at supersonic speed through the chamber of a silencing device attached to the muzzle of a cannon. The ensuing axisymmetric flow is calculated by using a TVD algorithm away from the bullet, in conjunction with the expanding cell scheme of chap. 4. Although the flow is unsteady, by examining the features of the bow shock wave created by passage of the bullet, one is able to assess, in some degree, the effectiveness of the projectile movement algorithm.

\subsection{The Euler Equations}

For axisymmetric flow problems, the Euler equations of gas dynamics can be written in strong conservation form as [3]

$$
\frac{\partial Q}{\partial t}+\frac{\partial F(Q)}{\partial X}+\frac{\partial G(Q)}{\partial Y}+W(Q)=0
$$

where

$$
Q=\left[\begin{array}{l}
\rho \\
m \\
n \\
e
\end{array}\right], \quad F=\left[\begin{array}{l}
\frac{m}{\rho}+P \\
m v \\
(e+P)
\end{array}\right], G=\left[\begin{array}{l}
n \\
n u \\
\frac{m^{2}}{\rho}+P \\
(e+P) \frac{n}{\rho}
\end{array}\right]
$$


and

$$
\mathrm{W}=\frac{\mathrm{n}}{\mathrm{Y}}\left[\begin{array}{c}
\mathrm{l} \\
\frac{\mathrm{m}}{\rho} \\
\frac{\mathrm{n}}{\rho} \\
\mathrm{e}+\mathrm{P} / \mathrm{o}
\end{array}\right]
$$

In these equations, $\rho$ is the density; $m=\rho u, n=\rho v$, are the components of momentum, and $u, v$ are velocities in the $x-$ and $\mathrm{y}$-directions, respectively. $\mathrm{P}$ is the pressure, and $e=\rho E$, where $E$ is the specific total energy, related to the specific internal energy $\bar{e}$, by

$$
E=\bar{e}+\frac{u^{2}+v^{2}}{2}
$$

The equation of state is

$$
P=(\gamma-1) \rho \bar{e},
$$

where $r$ is the ratio of specific heats.

\subsection{Eigenvector Projection}

The scheme of Harten referred to here is a 
second-order accurate extension of the Roe scheme (view 2) previously discussed. It is well documented in references $[6,19,20]$. Consider the application of Harten's method to an equation

$$
\frac{\partial Q}{\partial t}+\frac{\partial \hat{F}}{\partial \xi}(Q)=0
$$

where $\hat{F}(Q)$ has the Jacobian matrix

$$
\hat{A}=\xi_{X^{A}}+\xi_{Y^{B}}
$$

with $A=\frac{\partial F}{\partial Q}$ and $B=\frac{\partial G}{\partial Q}$ the Jacobian matrices of $F$ and $G$ [20]. The choices $\xi=\mathrm{x}$ or $\xi=\mathrm{y}$ then yield, respectively, the one-dimensional equation necessary for the $\mathrm{x}$-sweep or $\mathrm{y}$-sweep, as utilized in calculations which employ the technique of operator splitting $[11,21]$. Let $c$ be the local speed of sound; then the eigenvalues of $\hat{A}$ are [22]

$$
\left(a^{1}, a^{2}, a^{3}, a^{4}\right)=(\hat{\bar{U}}-c, \hat{\bar{U}}, \hat{\bar{U}}+c, \hat{\bar{U}})
$$

where 


$$
\hat{\bar{u}}=\frac{\xi_{x} u+\xi_{y} v}{\sqrt{\xi_{x}^{2}+\xi_{y}^{2}}}=k_{1} u+k_{2} v
$$

Furthermore, let $R=\left(R^{1}, R^{2}, R^{3}, R^{4}\right)$ be the matrix whose columns are the eigenvectors of $\hat{A}$. A choice of $R$ and $R^{-1}$ [20] can be written

$R=\left[\begin{array}{cccc}I & 1 & 1 & 0 \\ u-k_{1} c & u & u+k_{1} c & k_{2} \\ v-k_{2} c & v & v+k_{2} c & -k_{1} \\ H-k_{1} u c-k_{2} v c & \frac{u^{2}+v^{2}}{2} & H+k_{1} u c+k_{2} v c & k_{2} u-k_{1} v\end{array}\right]$

where $k_{1}, k_{2}$ are determined from eqn. (5.7);

$$
\mathrm{H}=\frac{\mathrm{c}^{2}}{\mathrm{r}-1}+\frac{\mathrm{u}^{2}+\mathrm{v}^{2}}{2}
$$

and the elements $z_{i j}$ of the matrix $R^{-1}$ are given by

$$
\begin{aligned}
& z_{11}=1 / 2\left(b_{1}+k_{1} u / c+k_{2} v / c\right) \\
& z_{12}=-1 / 2\left(b_{2} u+k_{1} / c\right) \\
& z_{13}=-1 / 2\left(b_{2} v+k_{2} / c\right)
\end{aligned}
$$




$$
\begin{aligned}
& z_{14}=1 / 2 b_{2} \\
& z_{21}=1-b_{1} \\
& z_{22}=b_{2} u \\
& z_{23}=b_{2} v \\
& z_{24}=-b_{2} \\
& z_{31}=1 / 2\left(b_{1}-k_{1} u / c-k_{2} v / c\right) \\
& z_{32}=-1 / 2\left(b_{2} u-k_{1} / c\right) \\
& z_{33}=-1 / 2\left(b_{2} v-k_{2} / c\right) \\
& z_{34}=1 / 2 b_{2} \\
& z_{41}=-k_{2} u+k_{1} v \\
& z_{42}=k_{2} \\
& z_{43}=-k_{1} \\
& z_{44}=0
\end{aligned}
$$

with

$$
\begin{aligned}
& b_{1}=b_{2}\left(u^{2}+v^{2}\right) / 2 \\
& b_{2}=(\gamma-1) / c^{2}
\end{aligned}
$$

Let a uniform grid spacing $\Delta x, \Delta y$ be introduced, with

$$
x_{j}=j \cdot \Delta x, \quad y_{k}=k \cdot \Delta y
$$

Denote by $o_{j+1 / 2, k}$ some symmetric average (the Roe average [10] was used for computational experiments) of $Q_{j, k}$ and 
$Q_{j+1, k} \cdot$ Let $a_{j+1 / 2}^{e}, R_{j+1 / 2}, R_{j+1 / 2}^{-1}$ denote evaluations of $a^{l}, R, R^{-1}$ on the symmetric average $\ell_{j}+1 / 2, k$. We define

$$
\alpha_{j+1 / 2}=R_{j+1 / 2}^{-1}\left(Q_{j+1, k}-Q_{j, k}\right)
$$

as the component of

$$
\Delta_{j+1 / 2} Q=\left(Q_{j+1, k}-Q_{j, k}\right)
$$

in the e-th characteristic $\xi$-direction [6]. The vector $\alpha$ of eqn. (5.11) can be written as

$$
\left[\begin{array}{l}
a^{1} \\
\alpha^{2} \\
\alpha^{3} \\
\alpha^{4}
\end{array}\right]_{j+1 / 2}=\left[\begin{array}{c}
\frac{a a-b b}{2} \\
\Delta j+1 / 2^{\rho-a a} \\
\frac{a a+b b}{2} \\
c c
\end{array}\right]
$$

where

$$
\begin{aligned}
a a= & \frac{\gamma-1}{c_{j+1 / 2}^{2}}\left[\Delta_{j+1 / 2} e+\frac{u_{j+1 / 2}^{2}+v_{j+1 / 2}^{2}}{2} \Delta_{j+1 / 2} \rho\right. \\
& \left.-u_{j+1 / 2} \Delta_{j+1 / 2} m-v_{j+1 / 2} \Delta_{j+1 / 2} n\right]
\end{aligned}
$$




$$
\begin{aligned}
b b & =\frac{1}{c_{j+1 / 2}}\left[k_{1} \Delta_{j+1 / 2} m-\left(k_{1} u_{j+1 / 2}+k_{2} v_{j+1 / 2}\right) \Delta_{j+1 / 2} o\right. \\
& \left.+k_{2} \Delta_{j+1 / 2} n\right] \\
c c & =-k_{1} \Delta j+1 / 2^{n}-\left(k_{2} u_{j+1 / 2}-k_{1} v_{j+1 / 2} \Delta_{j+1 / 2}\right. \\
& +k_{2} \Delta_{j}+1 / 2^{m}
\end{aligned}
$$

with

$$
\Delta_{j+1 / 2} \rho=\rho_{j+1, k}-\rho_{j, k}, \Delta_{j+1 / 2} m=m_{j+1, k}-m_{j, k}(5.13 e)
$$

and

$\Delta_{j+1 / 2} n=n_{j+1, k}-n_{j, k}, \Delta_{j+1 / 2} e=e_{j+1, k}-e_{j, k}$

The simplest form for $Q_{j+1 / 2, k}$ is

$$
Q_{j+1 / 2, k}=\left(Q_{j+1, k}+Q_{j, k}\right) / 2
$$

Roe's form of the averaging in the $\xi$-direction is: 


$$
\begin{aligned}
u_{j+1 / 2, k} & =\frac{\bar{D} u_{j+1, k}+u_{j, k}}{\bar{D}+1} \\
v_{j+1 / 2, k} & =\frac{\bar{D} v_{j+1, k}+v_{j, k}}{\bar{D}+1} \\
H_{j+1 / 2, k} & =\frac{\bar{D} H_{j+1, k}+H_{j, k}}{\bar{D}+1} \\
c_{j+1 / 2, k}^{2} & =(\gamma-1)\left[H_{j+1 / 2, k}-1 / 2\left(u_{j+1 / 2, k}^{2}+v_{j+1 / 2, k}^{2}\right)\right] \\
\bar{D} & =\sqrt{\rho j+1, k / \rho j, k} \\
H & =\frac{\gamma P}{(\gamma-1) \rho}+\frac{1}{2}\left(u^{2}+v^{2}\right)
\end{aligned}
$$

Similariy, Roe's averaging can be obtained for $u_{j, k+1 / 2}$, $v_{j, k+1 / 2}$ and $c_{j, k+1 / 2}$. In the numerical experiments Roe's averaging is used. 
5.3 An Operator-Split, TVD Algorithm

Assume that operator splitting is to be employed in solving eqn. (5.1) on a uniform grid. The flux terms are updated by applying Harten's method individually to eqn. (5.4), with $\xi=x$ and $\xi=y$ respectively; a predictorcorrector method is applied to update source terms. The solution at time $\tau$ is advanced to time $t+2 \tau$ by application of the following sequence of operators:

$$
Q_{j, k}^{n+2}=L_{x} I_{y} I_{s} I_{s} I_{y} I_{x} Q_{j, k}^{n}
$$

where

$$
\begin{aligned}
& I_{x}: Q_{j, k}^{*}=Q_{j, k}^{n}-\frac{\Delta t}{\Delta x}\left(\hat{F}_{j+1 / 2, k}^{n}-\hat{F}_{j-I / 2, k^{n}}^{n}\right) \\
& I_{y}: Q_{j, k}^{* *}=Q_{j, k}^{*}-\frac{\Delta t}{\Delta y}\left(\hat{G}_{j, k+1 / 2}^{n}-\hat{G}_{j, k-1 / 2}^{n}\right) \\
& I_{s}: \bar{Q}_{j, k}=Q_{j, k}^{* *}-\Delta t W\left(Q_{j, k}^{* *}\right) \\
& Q_{j, k}^{n+1}=\left[Q_{j, k}^{*}+\bar{Q}_{j, k}-\Delta t w\left(\bar{Q}_{j, k}\right)\right] / 2
\end{aligned}
$$


The numerical flux $\hat{F}_{j+1 / 2, k}$ is given by:

$$
\begin{aligned}
& \hat{F}_{j+1 / 2, k}=\left[F\left(Q_{j, k}\right)+F\left(Q_{j+1, k}\right)\right. \\
& \left.+\frac{\Delta x}{\Delta t} \sum_{l=1}^{4} B_{j+1 / 2, k}^{e} R_{j+1 / 2, k}^{l}\right] / 2 \\
& B_{j+1 / 2, k}^{e}=g_{j, k}^{e}+g_{j+1, k}^{e} \\
& \text { - } T\left(v_{j+1 / 2, k}^{e}+\gamma_{j+1 / 2, k}^{e}\right) \alpha_{j+1 / 2, k}^{e} \\
& g_{j, k}^{e}=(1 / 8) s_{j+1 / 2, k}^{e} \max \left[0, \min \left(\mid \alpha_{j+1 / 2, k}^{e} 1, \alpha_{j-1 / 2, k}^{e} s_{j+1 / 2, k}^{e}\right)\right] \\
& s_{j+1 / 2, k}^{e}=\operatorname{sign}\left(\alpha_{j+1 / 2, k}^{e}\right) \\
& r_{j+1 / 2, k}^{e}=\left\{\begin{array}{ll}
\left(g_{j+1, k}^{2}-g_{j, k}^{2}\right) / \alpha_{j+1 / 2, k}^{2}, & \alpha_{j+1 / 2, k}^{2} \neq 0 \\
0 & \alpha_{j+1 / 2, k}^{e}=0
\end{array} \quad\right. \text { (5.18e) } \\
& { }^{e} j+1 / 2, k=\frac{\Delta t}{\Delta x} a_{j+1 / 2, k}^{e}
\end{aligned}
$$




$$
T(z)=z^{2}+\frac{1}{4}
$$

Subscript $j+1 / 2$ denotes a quantity evaluated on the (Roe) average state, as discussed in section 2 (see eqns. (5.11-5.15)). No artificial compression terms [18] have been included in the algorithm. Such terms can give nonphysical results in regions of flow expansion, necessitating complicated program switching or interactive processing in order to anticipate such occurrences.

The equations for numerical flux $\hat{G}_{j, k+1 / 2}$ may be obtained from eqn. (5.18) by replacing $F$ with $G, \Delta x$ with $\Delta y$, and $(j+1 / 2, k)$ indices with $(j, k+1 / 2)$ indices. When eqn. $(5.17 \mathrm{c})$ is applied at a point $y=0$, it becomes necessary to apply L'Hospital's rule in evaluating the source term, W, as $v, Y$ approach zero.

\subsection{Weapons Nomenclature}

The weapon considered here is a $25 \mathrm{~mm}$ cannon, whose bore length is $80 \mathrm{cal}$.; the internal length of the silencer chamber is $7.6 \mathrm{cal}$; the silencer exit hole is 1.14 cal. in diameter; the exit wall thickness is 0.7 cal.. The computational domain extends $1.7 \mathrm{cal}$. downstream of the silencer exit, and approximately 1.0 cal. into the bore (see Fig. 5.1). The flow is impulsively started as the back of the 


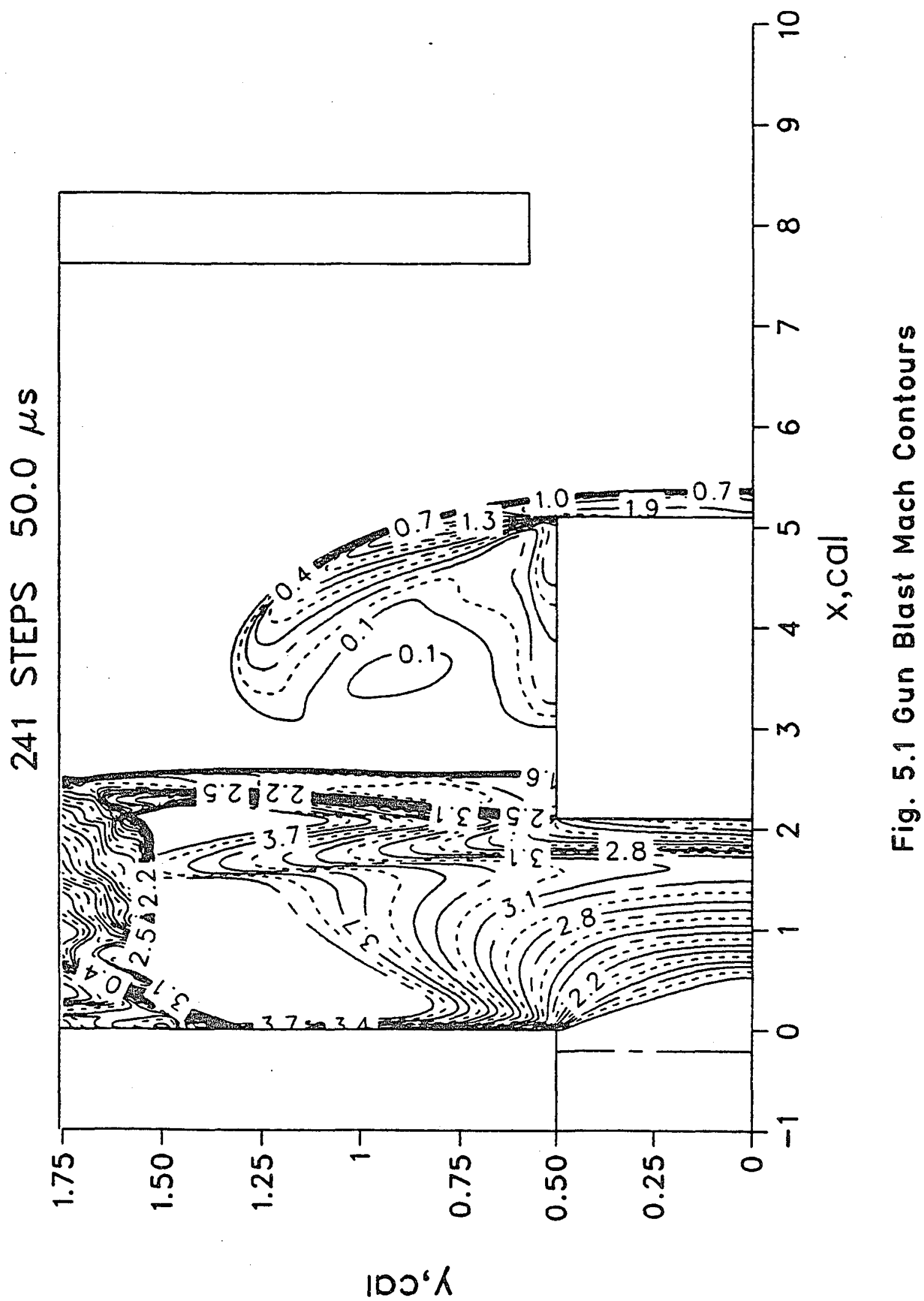


bullet, which is $3 \mathrm{cal}$. long, exits the bore. Upon exit, flow values at the muzzle were:

$$
\begin{aligned}
& \mathrm{P}_{e}=33.4 \mathrm{M} \mathrm{P} \\
& \mathrm{V}_{e}=1050 \mathrm{~m} / \mathrm{sec} \\
& \mathrm{M}_{e}=1.52
\end{aligned}
$$

Exterior to the muzzle atmospheric conditions prevail. As we are interested here in observing the bow shock formation in front of the moving projectile, no precursor flow is simulated. Although the flow around the projectile never becomes steady during the short duration of the calculation, a quasi-steady analysis of the bow shock allows the effectiveness of the bullet movement algorithm to be assessed.

5.5 Numerical Results; Supersonic Moving Projectile In this section we present numerical results from the TVD calculation discussed in secs. 5.1-5.4. Figures 5.1-5.6 show Mach number and density contour plots, as well as velocity vector plots, at respective times of 50 and $100 \mu \mathrm{sec}$ into the calculation. A mesh density of 81 points per calibre was used. Qualitatively, the results appear satisfactory. The expected features of such a flow problem are apparent: a bow shock forming in front of the projectile; a blast wave pushing from the rear; an inward 


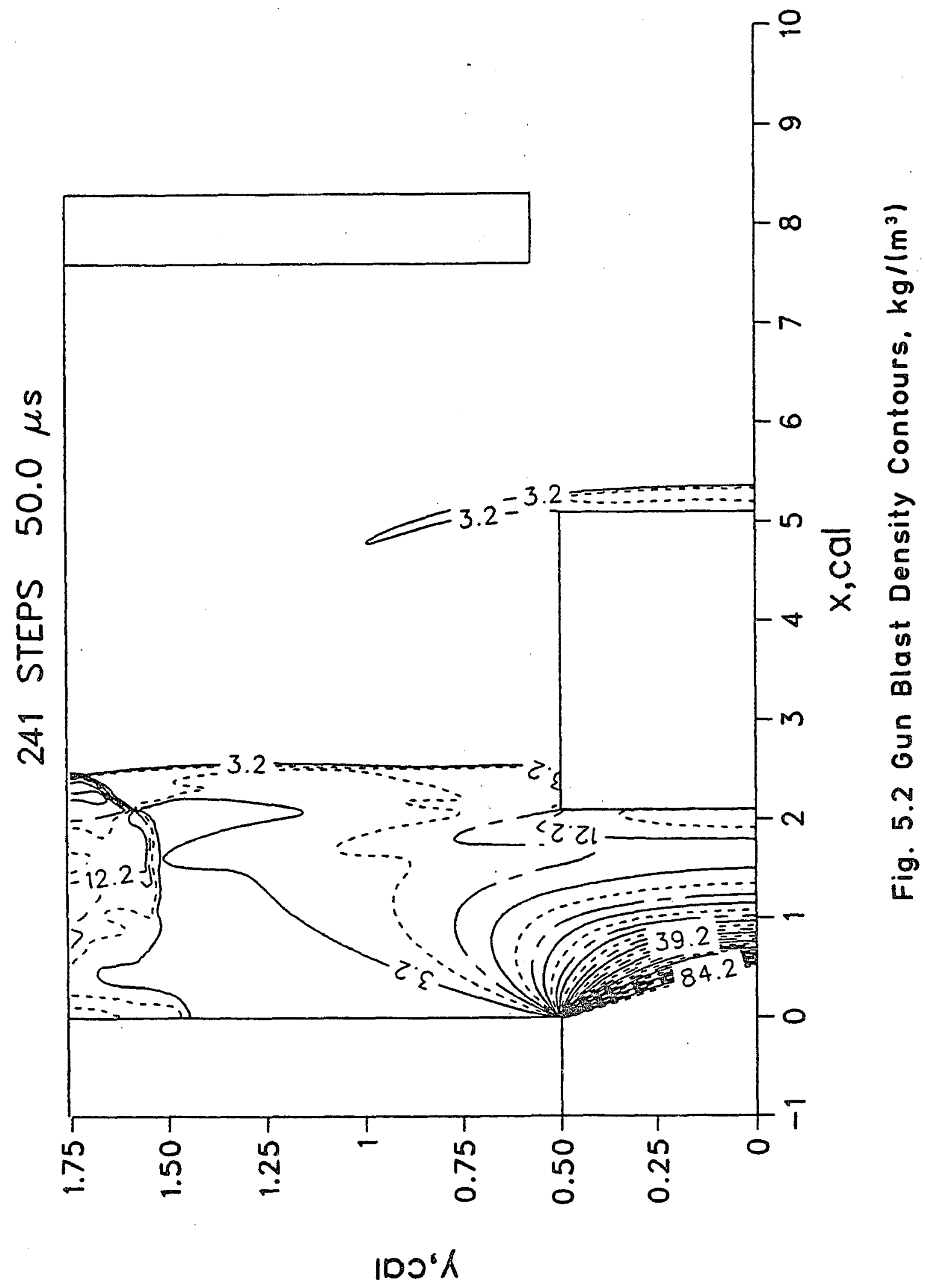




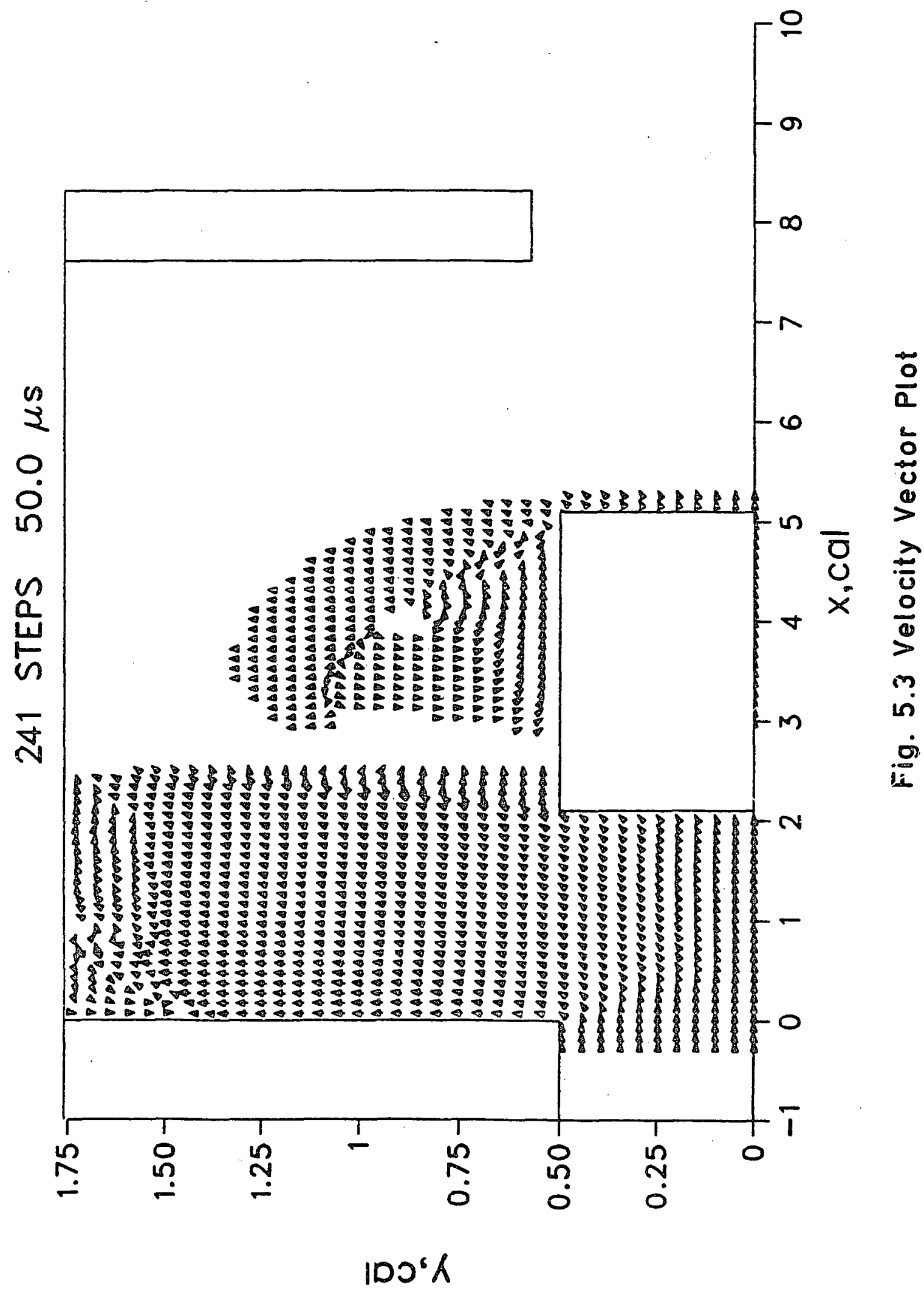




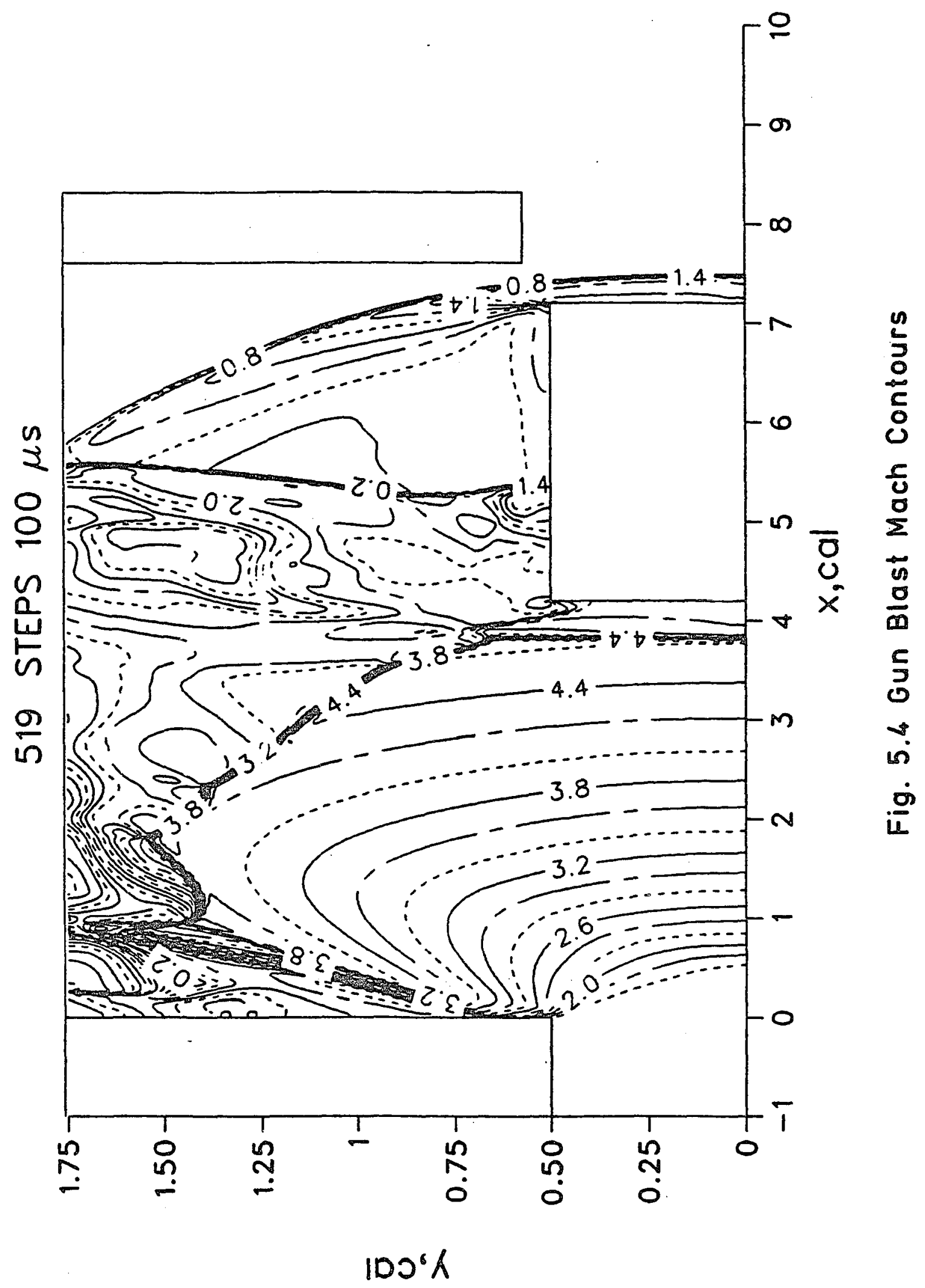




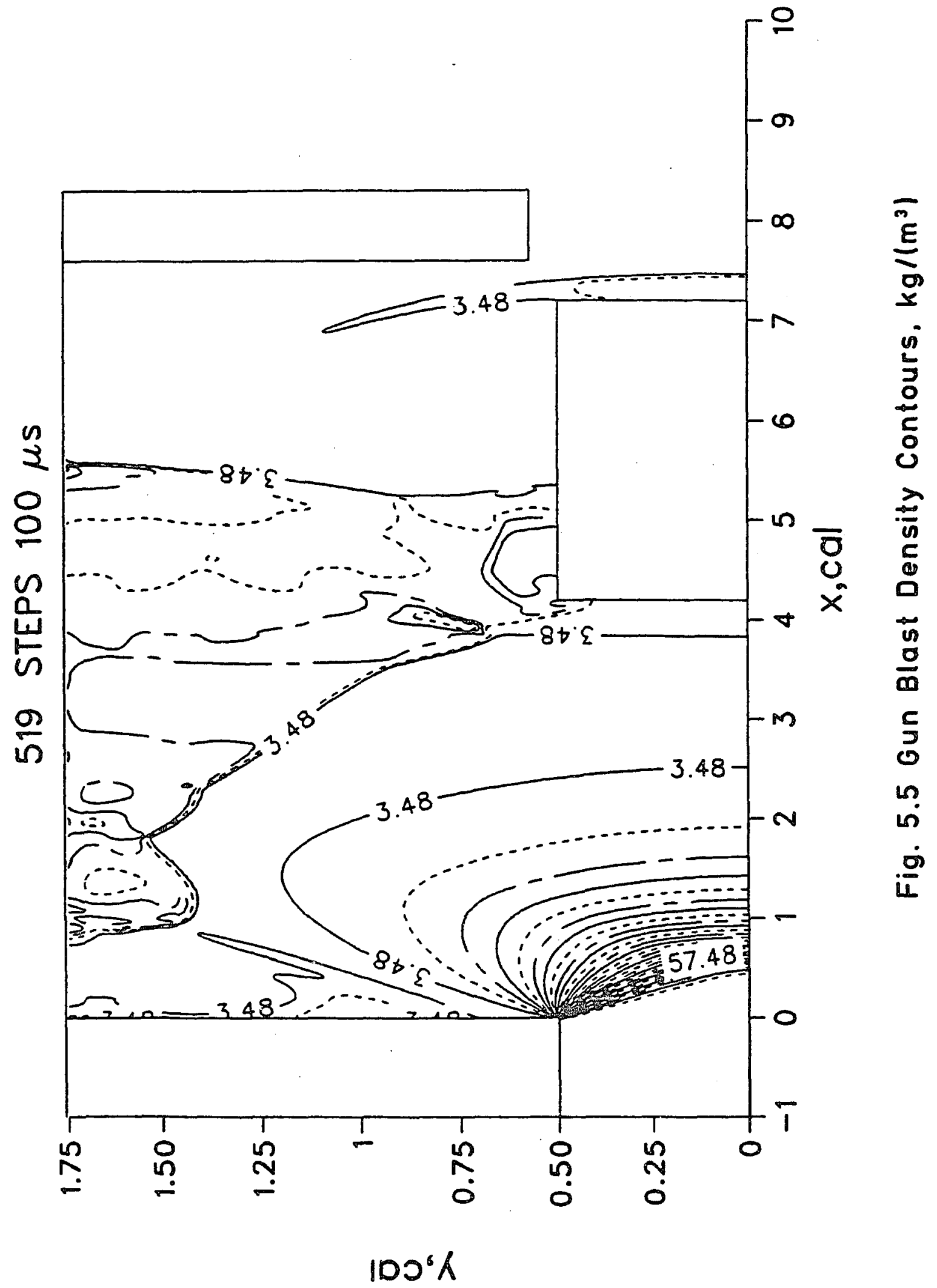




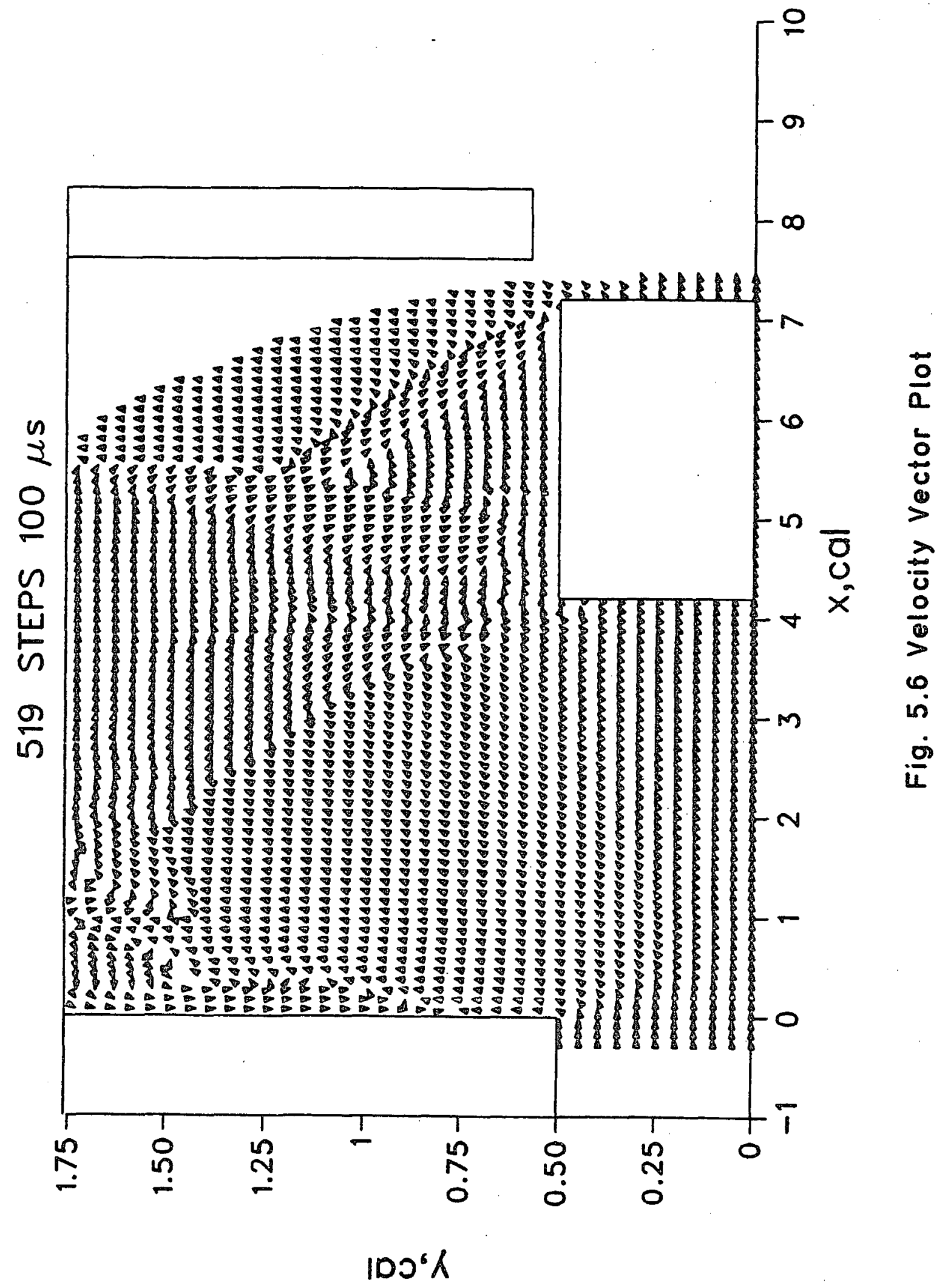


facing, recompression shock aft of the projectile; an expansion wave at the barrel exit, and recirculating flow in the corner and above the projectile front.

As further unsteadiness would result from the bullet passing through the silencer exit, the calculation was not extended past $100 \mu \mathrm{sec}$. During this time the flow through the bow shock appears to be approaching a steady state, although it is not completely steady. However, relative stagnation pressures, etc., at the body centerline can be compared with theoretical stagnation values for steady flow around a blunt body moving at the same speed as the projectile (see Table 5.1). In this fashion we assess the effectiveness of the bullet movement algorithm.

Table 5.1 indicates that stagnation pressure has essentially reached its steady value, at $100 \mathrm{usec}$. The fact that density has not yet become steady probably accounts for the larger differences between theoretical and calculated values for density and Mach number. Moreover, due to the effects of the noriuniform grid resulting from alternate larger or smaller lengths for the compressing cell at the projectile front, it is expected that complete steadiness probably can not be achieved. However, Table 5.1 shows that the method appears satisfactory, in accounting for the passage of the bullet through the flow. 
Table 5.1 Comparison of Stagnation Point Properties

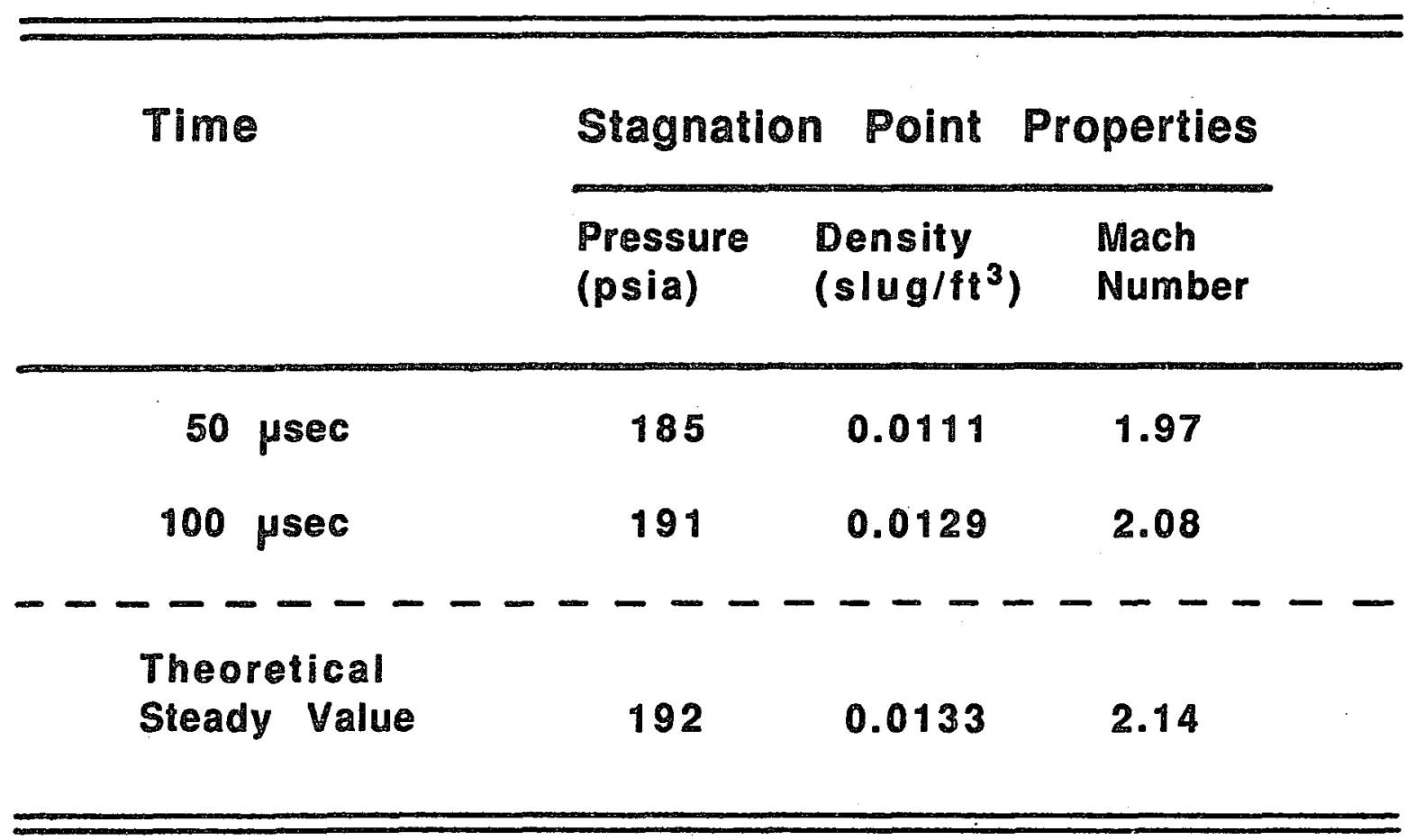


Chapter 6

A PHYSICALLY MOTIVATED WALL BOUNDARY TREATMENT

It has been pointed out by numerous investigators $[1,23]$ that an over-riding factor in the success or failure of a numerical experiment is the correctness of boundary condition treatment. For ideal compressible flow, vanishing of the normal component of velocity at a wall is the proper physical boundary condition. For the case of, say, a vertical wall, this condition reduces wall flux to the form

$$
\mathrm{F}_{\mathrm{W}}=\left[\begin{array}{l}
0 \\
\mathrm{P}_{\mathrm{W}} \\
0 \\
0
\end{array}\right],
$$

where the wall pressure, $P_{W}$, remains to be determined, in the application of cell-schemes such as that of Godunov [8] or Roe $[9,10]$.

We note that the most apparent remedy, extrapolation of wall pressure from the interior flow, makes no consideration of what physical processes may be occurring near the wall. For example, if flow at the grid point nearest to the wall is towards the wall, then locally a compression wave is expected to be advancing towards the wall; otherwise, a rarefaction wave is expected. The two 
situations lead, respectively, to cases where wall pressure is higher, or lower, than internal pressure. Moreover, extrapolation to the interior conceivably may not preserve the indicated pressure gradient at the wall, which leads to non-physical properties for the calculated flow.

The purpose of this chapter is to formulate an algorithm for determining wall pressure so as to be consistent with the local wave behavior. A modified boundary condition treatment of this type was employed in the numerical calculations previously cited in Chaps. 4-5; particularly, in the iteration process necessary to solve eqn. $(4.8)$.

\subsection{Rarefaction Waves}

When the flow is away from the wall, a rarefaction wave is expected. Consider again the Euler eqns. (4.1b); by transforming to the entropy variable, $s$, these equations can be equivalently written as [24]

$$
\frac{\partial \rho}{\partial t}+u \frac{\partial \rho}{\partial x}+\rho \frac{\partial u}{\partial x}=0
$$

$$
\frac{\partial u}{\partial t}+u \frac{\partial u}{\partial x}=-\frac{1}{\rho} \frac{\partial P}{\partial x}
$$




$$
\frac{\partial S}{\partial t}+u \frac{\partial S}{\partial x}=0
$$

We look for similarity solutions

$$
\begin{aligned}
& \mathrm{u}=\mathrm{u}(\xi) \\
& \rho=\rho(\xi) \\
& \mathrm{s}=\mathrm{s}(\xi)
\end{aligned}
$$

where

$$
\xi=\frac{x}{t}
$$

is the similarity variable. Now, eqns. (6.1c) and (6.2) imply that $s$ must be constant, or the flow is isentropic, if a similarity solution exists. Equations $(6.1 a, b)$ become

$$
\begin{aligned}
& (u-\xi) \frac{d \rho}{d \xi}+\rho \frac{d u}{d \xi}=0 \\
& (u-\xi) \frac{d u}{d \xi}+\frac{a^{2}}{\rho} \frac{d \rho}{d \xi}=0
\end{aligned}
$$

Non-trivial solutions of eqns. $(6.4-6.5)$ exist if and only 
if

$$
\xi=\frac{x}{t}=u \pm a
$$

where a is the local sound speed.

Consider now a quasi-one-dimensional treatment of flow near a wall boundary (see Fig. 6.1). At point B, which is the location of the grid point nearest to the wall, assume that flow is moving away from the wall in the negative $x$-direction.

For isentropic flow near the wall, eqn. (6.4) may be integrated to obtain

$$
u= \pm \frac{2}{\gamma-1}\left(a-a_{w}\right)
$$

as the relation between the wall point (where $u=0$ ) and near-wall flow velocity. Choosing the "+" sign in eqn. (6.7), there emerges

$$
a=a_{W}-\frac{1}{2}(\gamma-1)|u|
$$

Substituting eqn. (6.8) into the isentropic flow relation

$$
\frac{\rho}{\rho_{W}}=\left[\frac{a}{a_{W}}\right]^{\frac{2}{\gamma-1}}
$$


Legend:

Region I : a fixed wall

Region II : rarefaction wave

Region III : gas moving with constant velocity away from the wall
A : the wall boundary
$B$ : the nearest grid point to the wall

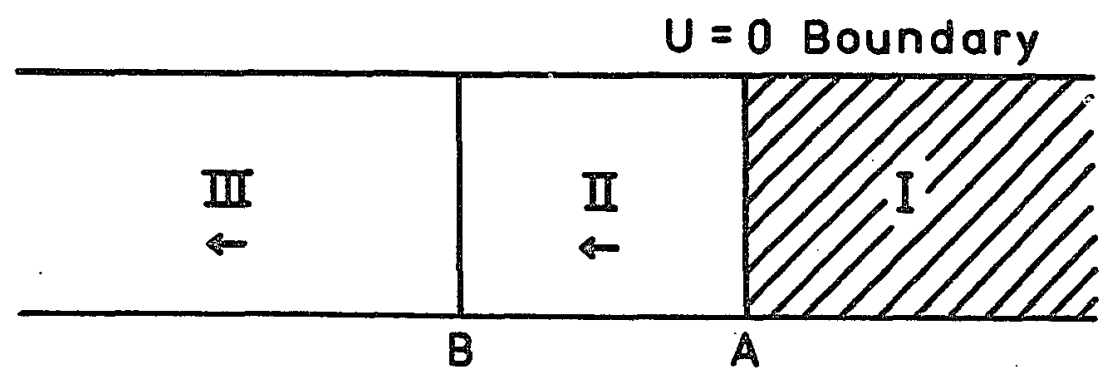

Fig. 6.1 Schematic of Rarefaction Waves 
yields

$$
\frac{\rho}{\rho_{\mathrm{W}}}=\left[1-\frac{1}{2}(\gamma-1) \frac{|\mathrm{u}|}{a_{\mathrm{W}}}\right]^{\frac{2}{\gamma-1}} .
$$

Thus, wall pressure and nearby flow pressure are related by

$$
P=P_{W}\left[1-\frac{1}{2}(\gamma-1) \frac{|u|}{a_{W}}\right]^{\frac{2 \gamma}{\gamma-1}}
$$

where $\gamma$ is the ratio of specific heats. For practical purposes, $a_{W} \simeq a$; hence, eqn. (6.11) allows wall pressure to be closely approximated, in a physical fashion.

\subsection{Compression Waves}

When the flow is towards the wall, a shock wave is assumed to connect the flow state at the wall with that at the nearest grid point. knowing all conditions on one side of the shock, and a single condition ( $u=0$ at the wall point) on the other side, allows the remaining state variables at the wall, and particularly wall pressure, to be determined from the usual shock jump relations. For a stationary normal shock, the Rankine-Hugoniot 
relations are [18]

$$
\begin{gathered}
\rho_{1} u_{1}=\rho_{2} u_{2} \\
P_{1}+\rho_{1} u_{1}^{2}=P_{2}+\rho_{2} u_{2}^{2} \\
\mathrm{~h}_{1}+u_{1}^{2} / 2=\mathrm{h}_{2}+u_{2}^{2} / 2
\end{gathered}
$$

Here, subscripts "1" and "2" represent the flow ahead or behind of the shock wave, respectively; and it is recalled that $h=e+P / \rho, E=e+u^{2} / 2$. Note that eqns. (6.12) also hold for gas velocities relative to the shock wave, whether or not the shock is moving or stationary. Now consider a normal shock moving at constant velocity $w$ into still air, and let the gas velocity behind the shock be $u$, where velocities are measured with respect to a fixed observer. Imagine that the observer moves with the shock, then the shock is fixed with respect to the observer; and the velocity of the gas ahead of the shock is $u_{1}=w$, while the velocity behind the shock is $u_{2}=w-u$. Therefore, eqns. $(6.12)$ can be written as

$$
\begin{aligned}
\rho_{1} W & =\rho_{2}(w-u) \\
P_{1}+\rho_{1} W^{2} & =P_{2}+\rho_{2}(w-u)^{2}
\end{aligned}
$$




$$
h_{1}+w^{2} / 2=h_{2}+(w-u)^{2} / 2
$$

In the case of a calorically perfect gas, there can be derived [18] from eqns. (6.13) the result

$$
u=\frac{a_{2}}{\gamma}\left(\frac{P_{2}}{P_{1}}-1\right)\left[\frac{\frac{2 \gamma}{\gamma+1}}{\frac{P_{2}}{P_{1}}+\frac{\gamma-1}{\gamma+1}}\right]^{\frac{1}{2}}\left[\frac{1+\frac{\gamma+1}{\gamma-1}\left(\frac{P_{2}}{P_{1}}\right)}{\frac{\gamma+1}{\gamma-1}\left(\frac{P_{2}}{P_{1}}\right)+\left(\frac{P_{2}}{P_{1}}\right)^{2}}\right]^{\frac{1}{2}}
$$

The pressure $P_{1}=P_{W}$ is the only unknown variable in eqn. (6.14). Let $x=\frac{P_{1}}{P_{2}}$, then eqn. $(6.14)$ can be rewritten as the quadratic equation:

$$
2 a_{2}^{2} x^{2}-\left[4 a_{2}^{2}+\gamma(\gamma+1) u^{2}\right] x+\left[2 a_{2}^{2}-\gamma(\gamma-1) u^{2}\right]=0
$$

whose solution is 


$$
x=\frac{\left[4 a_{2}^{2}+r(\gamma+1) u^{2}\right] \pm 4 r u \sqrt{\left[\frac{(\gamma+1) u^{2}}{4}\right]^{2}+a_{2}^{2}}}{4 a_{2}^{2}}
$$

since $x=\frac{P_{1}}{P_{2}}$ is always positive, choosing the "+" sign simplifies eqn. $(6.16)$ to

$$
x=I+\frac{\rho_{2}}{P_{2}} u \cdot v_{n}\left[I+\frac{\sqrt{v_{n}^{2}+\frac{\gamma P_{2}}{\rho_{2}}}}{v_{n}}\right]
$$

where

$$
v_{n}=\frac{(\gamma+1) u}{4}
$$

Therefore, the wall pressure $P_{W}=P_{2}=P_{2} \cdot x$ is determined. It is remarked that the wall boundary considered above is fixed. For the case of a wall moving at speed $u_{B}$ with absolute gas velocity $u_{g}$ behind the wall, eqns. (6.11) and (6.17) can be applied by employing the relative gas velocity $u=u_{g}-u_{B}$. 
Chapter 7

\section{SUMMARY AND CONCLUSIONS}

1. A local finite volume scheme wich accounts for projectile movement has been developed.

2. Results from numerical experiments in which the method is applied show it to be very effective, from the stand point of accuracy. The projectile movement problem appears effectively solved.

3. A weak derivative form (WDF) for linear hyperbolic systems of conservation laws has been derived. The utility of the WDF approach is that it shows how to finite difference in the presence of a moving discontinuity, without differencing across it.

4. A robust shock capturing algorithm results from the WDF approach. This algorithm can be readily extended to the nonlinear case, and it is shown to be equivalent to an upwind scheme of Roe. This leads to a better understanding of upwind differencing in general.

5. The upwind scheme of Roe is investigated in detail. Three separate views are obtained, one of which is new. It is shown that in the nonlinear case Roe's

\section{7}


scheme is equivalent to a matrix splitting scheme, and that this equivalence only holds in the case where the local linearization produces a Roe matrix characterized by property (W): (w) $\quad F(U)-F(V)=A(U, V)(U-V)$.

6. A boundary condition treatment considered effective in the case where a moving shock can strike a wall boundary has been discussed.

\section{Future Research Directions}

Further research motivated by the work discussed here concerns the development of higher order WDF forms of hyperbolic systems, which could be exploited to obtain higher order accurate shock capturing schemes. It would be of interest to determine whether Harten's second-order scheme can be derived by such an approach. 


\section{REFERENCES}

1. Moretti, G., "A Numerical Analysis of Muzzle Blast Precursor Flow," POIY M/AE Report No. 80-10, Brooklyn Polytechnic Institute, May 1980.

2. Cooke, C.H. and Fansler, K.N., "Comparison with Experiment for TVD Calculations of Blast Waves from A Shock Tube and A 105 MM Howitzer," TTCP Joint Workshop on Computation of Weapons Launch Blast Overpressure, $\mathrm{KTA}-7$, sponsored by The United States, Australia, Canada, and The United Kingdom. The Westin Hotel, Cincinnatti, Ohio, July 17, 1985.

3. Widhopf, G.F., Buell, J.C., and Schmidt, E.M., "Time-Dependent Near Field Muzzle Brake Flow simulations," AIAA-82-0973, AIAA/ASME Joint Thermophysics, Fluids, Plasma and Heat Transfer Conference, st. Louis, Missouri, June 7-12, 1982.

4. Carofano, G.C., U.S. Army Water-VIiet Arsenal, Water-Vliet, N.Y., private communication.

5. Fansler, K.N., "Gas Dynamic Quantities About A Ram Jet Projectile While in the Transitional

Ballistic Region," AIAA-85-18:0-CP, AIAA 12th Flight Mechanics Conference, Snowmass, Colorado, August 19-21, 1985.

6. Harten, A., "High Resolution Schemes for Hyperbolic Conservation Laws," J. Comp. Phys., Vol. 49, 1983, pp. 357-393.

7. Harten, A., Lax, P.D., and Van Leer, B., "On Upstream Differencing and Godunov-Type schemes for Hyperbolic Conservation Laws," SIAM Review, Vol. 25, No. 1, Jan. 1983, pp. 35-61.

8. Godunov, S.K., "A Difference scheme for Numerical Computation of Discontinuous Solutions of Equations of Fluid Dynamics," Math Sbornik, Vol. 47, 1959, pp. 271-306.

9. Roe, P.I., "The Use of the Riemann Problem in Finite Difference schemes," Proceedings 7th International Conference on Numerical Methods in Fluid Dynamics, Standford/NASA Ames, June 1980, Lecture Notes in Physics, 141, Springer-Verlag, New York, 1981, pp. 354-359. 
10. Roe, P.I., "Approximate Riemann Solvers, Parameter Vectors, and Difference Schemes," J. Comp. Phys. Vol. 43, 1981, PP. 357-372.

11. Steger, J.I. and Warming R.F., "Flux Vector splitting of the Inviscid Gasdynamic Equations with Application Finite-Difference Methods," J. Comp. Phys., Vol. 40, 1981, pp. 263-293.

12. Osher, S. and Solomon F., "Upwind Difference Schemes for Hyperbolic Systems of Conservation Laws,"

Math. Comp., Vol. 38, No. 158, 1982, pp. 339-374.

13. Van Leer, B., "On the Relation Between the UpwindDifferencing Schemes of Godunov, Engquist-osher, and Roe," ICASE Report No. 81-11, NASA Iangley Researach Center, Hampton, Virginia, March 16, 1981.

14. Courant, R. and Lax, P.D. "The Propagation of Discontinuities in Wave Motion," Proc. Nat. Acad. Sci., Vol. 42 , No. 11, 1956, pp. 872-876.

15. Chorin, A.J. and Marsden, J.E., A Mathematical Introduction to Fluid Mechanics, Springer-Verlag, New York, 1979.

16. Whitham, G.B., Iinear and Nonlinear Waves, Wiley, New York, 1974 .

17. Cooke, C.H. and Smith, P.W., "The Natural Occurrence of Flux Splitting in A Hyperbolic Setting," Numerical Methods for Partial Differential Equations, 1,1985 , pp. 1-11.

18. Anderson, J.D., Jr., Modern Compressible Flow with Historical Prespective, McGraw-Hill, New York, 1982.

19. Carofano, G., "Secondary Waves from Nozzle Blast," U.S. ARDC Technical Report No. ARLCB-TR-84028, Benet Weapons Laboratory, Watervliet, N.Y., 1984.

20. Yee, H.C. and Kutler, P., "Application of Second-order-Accurate Total Variation Diminishing (TVD) Schemes to the Euler Equations in General Geometries," NASA-TM-85845, Ames Research Center, Moffett Field, California, August 1983.

21. Cooke, C.H., "On Operator splitting of the Euler Equations Consistent with Harten's second-order Accurate TVD Scheme," Numerical Methods for Partial Differential Equations, 1,1985 , pp. 15-317. 
22. Warming, R.F., Beam, R., and Hyett, B.J.,

Diagonalization and Simultaneous symetrization of the Gas Dynamic Matrices," Math. of Comp., Vol. 29, No. 132, 1975, pp. 1037-1045.

23. Roache, P.J., Computational Fluid Dynamics, Hermosa Publishers, Alburquerque, N.M., 1972 .

24. Smoller, J., Shock Waves and Reaction-Diffusion Equations, Springer-Verlag, New York, 1983. 


\section{AUTOBIOGRAPHICAL STATEMENT}

Jen-Ing G. Hwang

\section{PLACE AND DATE OF BIRTH}

Taipei, Taiwan, Republic of China

January 11,1958

\section{EDUCATION}

M.S. in Computational and Applied Mathematics, May 1984

old Dominion University

Norfolk, Virginia

B.S. in Pure Mathematics

Minor in International Trade, June 1980

Fu Jen Catholic University

Taipei, Taiwan

Republic of China

\section{HONORS}

Phi Kappa Phi, National Honor Society

\section{PROFESSIONAI EXPERIENCE}

1. Research Assistant. (August 1985 to May 1987)

Department of Mathematics \& Statistics

old Dominion University

2. Teaching Assistant. (August 1982 to May 1985)

Department of Mathematics \& Statistics

old Dominion University

3. Teaching Assistant. (July 1980 to July 1982)

Computer Center

Fu Jen Catholic University 